\title{
GIT Quotients of Products of Projective Planes
}

\author{
Francesca Incensi (*)
}

ABSTRACT - We study the quotients for the diagonal action of $S L_{3}(\mathrm{C})$ on the $n$-fold product of $\mathrm{P}^{2}(\mathrm{C})$ : we are interested in describing how the quotient changes when we vary the polarization (i.e. the choice of an ample linearized line bundle). We illustrate the different techniques for the construction of a quotient, in particular the numerical criterion for semi-stability and the "elementary transformations" which are resolutions of precisely described singularities (case $n=6$ ).

\section{Introduction.}

Consider a projective algebraic variety $X$ acted on by a reductive algebraic group $G$. Geometric Invariant Theory (GIT) gives a construction of a $G$-invariant open subset $U$ of $X$ for which the quotient $U / / G$ exists and $U$ is maximal with this property (roughly speaking, $U$ is obtained from $X$ throwing away "bad" orbits). However the open $G$-invariant subset $U$ depends on the choice of a $G$-linearized ample line bundle. Given an ample $G$-linearized line bundle $L \in \operatorname{Pic}^{G}(X)$ over $X$, one defines the set of semi-stable points as

$$
X^{S S}(L):=\left\{x \in X \mid \exists n>0 \text { and } s \in \Gamma\left(X, L^{\otimes n}\right)^{G} \text { s.t. } s(x) \neq 0\right\},
$$

and the set of stable points as

$X^{S}(L):=\left\{x \in X^{S S}(L) \mid G \cdot x\right.$ is closed in $X^{S S}(L)$ and the stabilizer $G_{x}$ is finite $\}$.

Then it is possible to introduce a categorical quotient $X^{S S}(L) / / G$ in which two points are identified if the closure of their orbits intersect. Moreover as shown in [10], $X^{S S}(L) / / G$ exists as a projective variety and contains the orbit space $X^{S}(L) / G$ as a Zariski open subset.

(*) Indirizzo dell'A.: Dipartimento di Matematica, Università di Bologna, Piazza di Porta S. Donato, 5 - 40126 Bologna, Italy

E-mail: incensi@dm.unibo.it 


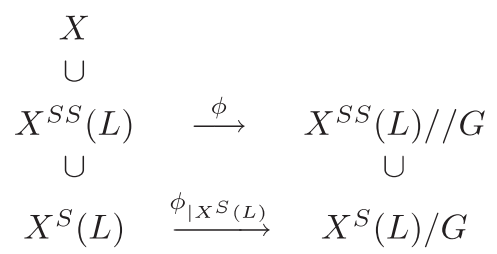

Question. - If one fixes $X, G$ and the action of $G$ on $X$, but lets the linearized ample line bundle $L$ vary in $\operatorname{Pic}^{G}(X)$, how do the open set $X^{S S}(L) \subset X$ and the quotient $X^{S S}(L) / / G$ change?

Dolgachev-Hu [4] and Thaddeus [11] proved that only a finite number of GIT quotients can be obtained when $L$ varies and gave a general description of the maps relating the various quotients.

In this paper we study the geometry of the GIT quotients for $X=\mathbb{P}^{2}(\mathrm{C}) \times \ldots \times \mathbb{P}^{2}(\mathrm{C})=\mathbb{P}^{2}(\mathrm{C})^{n}$. We give examples for $n=5$ and $n=6$. The contents of the paper are more precisely as follows.

Section 1 treats the general case $X=\mathbb{P}^{2}(C)^{n}$ : first of all the numerical criterion of semi-stability is proved (Proposition 1.1). By means of this it is possible to show that only a finite number of quotients $X^{S S}(m) / / G$ exists (Subsection 1.2). At the end of the section we introduce the elementary transformations which relate the different quotients.

Section 2 is concerned with the case $n=5$. Theorem 2.8 contains the main result of Section 2: we show that there are precisely six different quotients.

Section 3 discusses the case $n=6$ : the main results of this Section are concerned with the number of different geometric quotients that may be obtained (it is less than or equal to 38: Table 3.1) and with the singularities that may appear in the quotients. In particular there are only two different types of singularities: in Subsection 3.2 they are described, using the Étale Slice theorem. Theorem 3.2 collects these results. At the end of the Section two examples show how these singularities are resolved by "crossing the wall".

\section{The general case $X=\mathbb{P}^{2}(C)^{n}$.}

Let $G$ be the group $S L_{3}(\mathrm{C})$ acting on the variety $X=\mathbb{P}^{2}(\mathrm{C})^{n}$ and let $\sigma$ be the diagonal action 


$$
\begin{array}{rrrrr}
\sigma: G \quad \times & \mathbb{P}^{2}(\mathbb{C})^{n} & \rightarrow & \mathbb{P}^{2}(\mathbb{C})^{n} \\
g & , \quad\left(x_{1}, \ldots, x_{n}\right) & \mapsto & \left(g x_{1}, \ldots, g x_{n}\right)
\end{array}
$$

A line bundle $L$ over $X$ is determined by $L=L(m):=L\left(m_{1}, \ldots, m_{n}\right)=$ $=\bigotimes_{i=1}^{n} \pi_{i}^{*}\left(\mathcal{O}_{\mathbb{P}^{2}(\mathbb{C})}\left(m_{i}\right)\right), m_{i} \in Z \forall i$, where $\pi_{i}: X \rightarrow \mathbb{P}^{2}(\mathrm{C})$ is the $i$-th projection. In particular $L$ is ample iff $m_{i}>0, \forall i$.

Moreover since each $\pi_{i}$ is an $G$-equivariant morphism, $L$ admits a canonical $G$-linearization:

$$
\operatorname{Pic}^{G}(X) \cong Z^{n} .
$$

Thus a polarization is completely determined by the line bundle $L$.

Recall that a point $x \in X$ is said to be semi-stable with respect to the polarization $m$ iff there exists a $G$-invariant section of some positive tensor power of $L, \gamma \in \Gamma\left(X, L^{\otimes k}\right)^{G}$, such that $\gamma(x) \neq 0$. A semi-stable point is stable if its orbit is closed and has maximal dimension. The categorical quotient of the open set of semi-stable points exists and is denoted by $X^{S S}(m) / / G$ :

$$
X^{S S}(m) / / G \cong \operatorname{Proj}\left(\bigoplus_{k=0}^{\infty} \Gamma\left(X, L^{\otimes k}\right)^{G}\right) .
$$

The open set $X^{S}(m) / G$ of $X^{S S}(m) / / G$ is a geometric quotient.

We set $X^{U S}(m)=X \backslash X^{S S}(m)$, the closed set of unstable points and $X^{S S S}(m)=X^{S S}(m) \backslash X^{S}(m)$, the set of strictly semi-stable points.

\section{1 - Numerical Criterion of semi-stability.}

After fixing a polarization $L(m)$, we want to describe the set of semistable points $X^{S S}(m)$ : using the Hilbert-Mumford numerical criterion, we prove the following

Proposition 1.1. Let $x \in X$ and $|m|:=\sum_{i=1}^{n} m_{i}$. Then we have

$$
x \in X^{S S}(m) \Leftrightarrow\left\{\begin{array}{l}
\sum_{k, x_{k}=y} m_{k} \leq \frac{|m|}{3} \\
\sum_{j, x_{j} \in r} m_{j} \leq 2 \frac{|m|}{3}
\end{array}\right.
$$

for every point $y \in \mathbb{P}^{2}(C)$ and for every line $r \subset \mathbb{P}^{2}(C)$. 
Proof. Fixing projective coordinates on the $i$-th copy of $\mathbb{P}^{2}(\mathrm{C})$, $\left[x_{i 0}: x_{i 1}: x_{i 2}\right]$, a point $x \in X\left(\subset \mathbb{P}\left(\Gamma(X, L(m))^{*}\right)=\mathbb{P}^{N}(\mathrm{C})\right)$, is described by homogeneous coordinates of this kind:

$$
\prod_{i=1}^{n} x_{i 0}^{j_{i}} x_{i 1}^{k_{i}} x_{i 2}^{m_{i}-\left(j_{i}+k_{i}\right)}
$$

where $0 \leq j_{i}, k_{i} \leq m_{i}, j_{i}+k_{i} \leq m_{i}$.

Let $\lambda_{\alpha_{0}, \alpha_{1}, \alpha_{2}}$ be the one-parameter subgroup of $G$ defined by $\lambda_{\alpha_{0}, \alpha_{1}, \alpha_{2}}(t)=$ $=\operatorname{diag}\left(t^{\alpha_{0}}, t^{\alpha_{1}}, t^{\alpha_{2}}\right)$ where $\alpha_{0}+\alpha_{1}+\alpha_{2}=0$; we can assume $\alpha_{0} \geq \alpha_{1} \geq \alpha_{2}$.

The subgroup $\lambda_{\alpha_{0}, \alpha_{1}, \alpha_{2}}$ acts on every component of $\mathbb{C}^{N+1}$, multiplying by

$$
t^{\alpha_{0}} \sum_{i} j_{i}+\alpha_{1} \sum_{i} k_{i}+\alpha_{2} \sum_{i}\left(m_{i}-\left(j_{i}+k_{i}\right)\right) .
$$

By the definition of the numerical function of Hilbert-Mumford $\mu_{L}(x, \lambda)$, we are interested in determining the minimum value of

$$
\alpha_{0} \sum_{i=1}^{n} j_{i}+\alpha_{1} \sum_{i=1}^{n} k_{i}+\alpha_{2} \sum_{i=1}^{n}\left(m_{i}-\left(j_{i}+k_{i}\right)\right) .
$$

This should be obtained when $j_{i}=k_{i}=0, \forall i$; but if there are some $x_{i 2}=0$, then the minimum value becomes:

$$
\alpha_{2} \sum_{i, x_{i 2} \neq 0} m_{i}+\alpha_{1} \sum_{j, x_{j 2}=0, x_{j 1} \neq 0} m_{j}+\alpha_{0} \sum_{k, x_{k 2}=x_{k 1}=0} m_{k} .
$$

Thus $x \in X$ is semi-stable for the action of $\lambda_{\alpha_{0}, \alpha_{1}, \alpha_{2}}$ if and only if expression (2) is less than or equal to zero.

Let

$$
\alpha_{0}=\beta_{0}+\beta_{1}, \quad \alpha_{1}=-\beta_{0}, \quad \alpha_{2}=-\beta_{1} ;
$$

it follows that $\beta_{1} \geq-2 \beta_{0}, \beta_{1} \geq \beta_{0}$ e $\beta_{1} \geq 0$.

The expression (2) can be rewritten and the condition for semistability is

$$
\beta_{0}\left(\sum_{k, x_{k 2}=x_{k 1}=0} m_{k}-\sum_{j, x_{j 2}=0, x_{j 1} \neq 0} m_{j}\right)+\beta_{1}\left(\sum_{k, x_{k 2}=x_{k 1}=0} m_{k}-\sum_{i, x_{i 2} \neq 0} m_{i}\right) \leq 0
$$

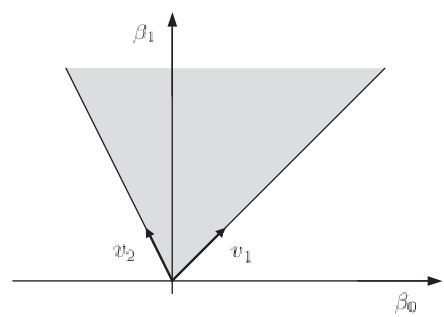

Fig. 1. - Plane $\beta_{0}, \beta_{1}$. 
The figure 1 shows that every couple $\left(\beta_{0}, \beta_{1}\right)$ that satisfies (3) is a positive linear combination of $v_{1}=(1,1)$ e $v_{2}=(-1,2)$. Thus the relation (3) must be verified in the two cases $\beta_{0}=\beta_{1}=1$ e $\beta_{0}=-1, \beta_{1}=2$. After a few calculations we obtain that $x$ is semi-stable for the action of all $\lambda_{\alpha_{0}, \alpha_{1}, \alpha_{2}}$ if and only if

$$
\begin{cases}\sum_{h, x_{h}=y} m_{h} \leq|m| / 3, & y \in \mathbb{P}^{2}(\mathbb{C}) \\ \sum_{l, x_{l} \in r} m_{l} \leq 2|m| / 3, & r \subset \mathbb{P}^{2}(\mathbb{C})\end{cases}
$$

for $y=[1: 0: 0]$ and $r$ the line $x_{2}=0$. Since every one-parameter subgroup is conjugate to one of the form $\lambda_{\alpha_{0}, \alpha_{1}, \alpha_{2}}$ the proposition follows.

REMARK 1.2. The case with all $m_{i}=1$ is a special case of [10] Proposition 4.3 .

REMARK 1.3. $x \in X^{S}(m)$ iff the numerical criterion (1) is verified with strict inequalities.

The numerical criterion can be restated as follows: if $K$ and $J$ are subsets of $\{1, \ldots, n\}$, then we can associate them with the numbers:

$$
\gamma_{K}^{C}(m)=|m|-3 \sum_{k \in K} m_{k}, \quad \gamma_{J}^{L}(m)=2|m|-3 \sum_{j \in J} m_{j} .
$$

In particular we have: $\gamma_{J}^{C}(m)=-\gamma_{J^{\prime}}^{L}(m)$; where $J^{\prime}=\{1, \ldots, n\} \backslash J$.

Now for every collection of disjoint subsets $K_{1}, \ldots, K_{r}$ of $\{1, \ldots, n\}$ with $\left|K_{l}\right| \geq 2$, we consider the set of configurations $\left(x_{1}, \ldots, x_{n}\right)$ where the points indexed by each set $K_{l}$ are coincident and there are no further coincidences:

$$
U_{K_{1}, \ldots, K_{r}}^{C}=\left\{x \in X \mid \text { if } i \neq j, \text { then } x_{i}=x_{j} \Leftrightarrow i, j \in K_{l} \text { for some } l\right\} .
$$

We write also

$$
U_{\emptyset}^{C}=\left\{x \in X \mid x_{i} \neq x_{j} \text { if } i \neq j\right\}
$$

In the same way, for every collection of subsets $J_{1}, \ldots, J_{s}$ of $\{1, \ldots, n\}$ with $\left|J_{l}\right| \geq 3$ and $J_{l} \nsubseteq J_{p}$ if $l \neq p$, define

$$
U_{J_{1}, \ldots, J_{s}}^{L}=\left\{\begin{array}{ll}
x \in X \mid & \text { if } i, j, k \text { are distinct, then } \\
& x_{i}, x_{j}, x_{k} \text { are collinear } \Leftrightarrow i, j, k \in J_{l} \text { for some } l
\end{array}\right\} .
$$


Here by "collinear" we mean that there exists a line $r$ containing $x_{i}, x_{j}, x_{k}$; we do not require that these points be distinct.

These definitions have the effect that the subsets $U_{K_{1}, \ldots, K_{r}}^{C} \cap U_{J_{1}, \ldots, J_{s}}^{L}$ correspond to points having precisely specified sets of coincident and collinear points. Note that the points of the subsets $U_{K_{1}, \ldots, K_{r}}^{C}$ have necessarily some "implied collinearities" (for example, if $x_{1}=x_{2}$ then $x_{1}, x_{2}, x_{3}$ are collinear). It will be convenient to write $V_{K_{1}, \ldots, K_{r}}^{C}$ for the subset of $U_{K_{1}, \ldots, K_{r}}^{C}$ consisting of points for which there are no non-implied collinearities. We write also

$$
V_{J_{1}, \ldots, J_{s}}^{L}=U_{J_{1}, \ldots, J_{s}}^{L} \cap U_{\emptyset}^{C}
$$

for the set of points with collinearities given by $J_{1}, \ldots, J_{s}$ and no coincidences.

REMARK 1.4. We have $U_{K_{1}, \ldots, K_{r}}^{C} \cap U_{J_{1}, \ldots, J_{s}}^{L} \subseteq X^{S S}(m)$ if and only if $m_{i} \leq \frac{|m|}{3}$ for all $i, \quad \gamma_{K_{l}}^{C}(m) \geq 0$ for $1 \leq l \leq r, \quad \gamma_{J_{l}}^{L}(m) \geq 0$ for $1 \leq l \leq s$.

Moreover, if any of these inequalities fails, then

$$
U_{K_{1}, \ldots, K_{r}}^{C} \cap U_{J_{1}, \ldots, J_{s}}^{L} \cap X^{S S}(m)=\emptyset .
$$

The same holds for $X^{S}(m)$ if we replace all inequalities by strict inequalities. In view of this, when studying $X^{S S}(m)$ and $X^{S}(m)$, it is sufficient to consider the subsets $U_{K}^{C} \cap U_{J}^{L}$ or even $V_{K}^{C}$ and $V_{J}^{L}$. In fact

$$
V_{K}^{C} \subseteq X^{S S}(m) \Leftrightarrow m_{i} \leq \frac{|m|}{3} \text { for all } i \text { and } \gamma_{K}^{C}(m) \geq 0,
$$

and

$$
V_{J}^{L} \subseteq X^{S S}(m) \Leftrightarrow m_{i} \leq \frac{|m|}{3} \text { for all } i \text { and } \gamma_{J}^{L}(m) \geq 0,
$$

with similar statements for $X^{S}(m)$.

\section{2 - Quotients.}

Proposition 1.5. Let

$$
U^{G E N}:=\left\{x \in X \mid x_{1}, \ldots, x_{n} \text { in general position }\right\} \subset X,
$$

(i.e. every four points among $\left\{x_{1}, \ldots, x_{n}\right\}$ are a projective system of 
$\left.\mathbb{P}^{2}(\mathbb{C})\right)$. Then

1. $X^{S S}(m) \neq \emptyset \Leftrightarrow U^{\mathrm{GEN}} \subset X^{S S}(m) \Leftrightarrow m_{i} \leq \frac{|m|}{3}$ for all $i$

2. $X^{S}(m) \neq \emptyset \Leftrightarrow U^{G E N} \subset X^{S}(m) \Leftrightarrow m_{i}<\frac{|m|}{3}$ for all $i$;

Moreover, if $n \geq 5$,

$$
X^{S}(m) \neq \emptyset \Leftrightarrow \operatorname{dim}\left(X^{S S}(m) / / G\right)=2(n-4) .
$$

Proof. Except for the final statement, this follows from Remark 1.4. Since $X^{S}(m) / G$ is a geometric quotient, it is obvious that $X^{S}(m) \neq \emptyset \Rightarrow$ $\Rightarrow \operatorname{dim}\left(X^{S S}(m) / / G\right)=2(n-4)$. On the other hand, if $X^{S}(m)=\emptyset$ but $X^{S S}(m) \neq \emptyset$, we must have $m_{i}=|m| / 3$ for some $i$. We can suppose without loss of generality that $i=1$. Every orbit in $U^{\mathrm{GEN}}$ contains a point of the form

$$
\left(\begin{array}{cccccc}
1 & 0 & 0 & 1 & \ldots & a \\
0 & 1 & 0 & 1 & \ldots & b \\
0 & 0 & 1 & 1 & \ldots & c
\end{array}\right)
$$

with $a, b, c \neq 0$. Acting by the one parameter subgroup $\lambda_{2,-1,-1}$ and letting $t \rightarrow 0$, we obtain

$$
\left(\begin{array}{cccccc}
1 & 0 & 0 & 0 & \ldots & 0 \\
0 & 1 & 0 & 1 & \ldots & b \\
0 & 0 & 1 & 1 & \ldots & c
\end{array}\right)
$$

This point belongs to the closure of the original orbit and remains semistable. It follows that

$$
X^{S S}(m) / / G \cong\left(\left(\mathbb{P}^{1}(\mathbb{C})\right)^{n-1}\right)^{S S}\left(m_{2}, \ldots, m_{n}\right),
$$

which has dimension less than or equal to $n-4$.

We know that the quotient $X^{S S}(m) / / G$ depends on the choice of the polarization $L(m)$ : moreover Dolgachev-Hu [4] and Thaddeus [11] have proved that when $L(m)$ varies, then there exists only a finite number of different quotients.

Now we give a proof of the same result in our case.

COROLlary 1.6. There are finitely many different quotients $X^{S S}(m) / / G$. 
PRoof. It follows from Proposition 1.5 and Remark 1.4 that

$$
X^{S S}(m)=U^{\mathrm{GEN}} \cup \mathcal{U}^{S S}(m),
$$

where $\mathcal{U}^{S S}(m):=\bigcup\left\{U_{K_{1}, \ldots, K_{r}}^{C} \cap U_{J_{1}, \ldots, J_{s}}^{L} \mid U_{K_{1}, \ldots, K_{r}}^{C} \cap U_{J_{1}, \ldots, J_{s}}^{L} \subset X^{S S}(m)\right\}$. In particular we can construct only a finite number of different sets $\mathcal{U}^{S S}(m)$ and as a consequence there exists a finite number of different open sets $X^{S S}(m)$; in conclusion only a finite number of quotients $X^{S S}(m) / / G$ exists.

REMARK 1.7. If $n \leq 3$, then $X^{S}(m)=\emptyset$; moreover $X^{S S}(m)=\emptyset$ except when $n=3$ and $m_{1}=m_{2}=m_{3}$, in which case $X^{S S}(m)=U^{\mathrm{GEN}}$ and $X^{S S}(m) / / G$ is a point. If $n=4$ and $m_{i}<|m| / 3$ for all $i$, then $X^{S}(m)=$ $=X^{S S}(m)=U^{\mathrm{GEN}}$ and $X^{S}(m) / G$ is a point. Otherwise $X^{S}(m)=\emptyset$ and either $X^{S S}(m)=\emptyset$ or $X^{S S}(m) / / G$ is a point.

\section{3 - Elementary transformations.}

Let $m$ be a polarization such that 3 divides $|m|$ and $X^{S}(m) \neq \emptyset, X^{S}(m) \subsetneq X^{S S}(m)$; let us consider "variations" of $m$ as follows:

$$
\widehat{m}=m(0, \ldots, 0, \underbrace{1}_{i}, 0, \ldots, 0) .
$$

We can have two different kind of variations, depending on the value $|\widehat{m}|$ :

1. $\widehat{m} \stackrel{+1_{i}}{\longrightarrow} m \quad$ (i.e. $|\widehat{m}| \equiv 2 \bmod 3$ );

2. $\widehat{m} \stackrel{-1_{i}}{\longrightarrow} m \quad$ (i.e. $\left.|\widehat{m}| \equiv 1 \bmod 3\right)$.

In both cases we have $X^{S}(\widehat{m})=X^{S S}(\widehat{m})$; studying the relations between values $\gamma_{J}^{C}(\widehat{m}), \gamma_{K}^{L}(\widehat{m})$ and values $\gamma_{J}^{C}(m), \gamma_{K}^{L}(m)$, we observe that

1. $\widehat{m} \stackrel{+1_{i}}{\longrightarrow} m$

$$
\begin{aligned}
& X^{S}(\widehat{m}) \subset X^{S S}(m), \quad X^{S}(\widehat{m})=X^{S S}(m) \backslash \bigcup_{i \notin J, \gamma_{J}^{C}(m)=0 \vee \gamma_{J}^{L}(m)=0} V_{J}^{*}, \\
& X^{S}(m) \subset X^{S}(\widehat{m}), \quad X^{S}(m)=X^{S}(\widehat{m}) \backslash \bigcup_{i \in H, \gamma_{H}^{C}(\widehat{m})=2 \vee \gamma_{H}^{L}(\widehat{m})=1} V_{H}^{*},
\end{aligned}
$$

where $V_{J}^{*}$ is $V_{J}^{C}$ if $\gamma_{J}^{C}(m)=0$ or $V_{J}^{L}$ if $\gamma_{J}^{L}(m)=0$ and in the same way $V_{H}^{*}$ is $V_{H}^{C}$ if $\gamma_{H}^{C}(\widehat{m})=2$ or $V_{H}^{L}$ if $\gamma_{H}^{L}(\widehat{m})=1$. 
2. $\widehat{m} \stackrel{-1_{i}}{\longrightarrow} m$

$$
\begin{aligned}
& X^{S}(\widehat{m}) \subset X^{S S}(m), \quad X^{S}(\widehat{m})=X^{S S}(m) \backslash \underset{i \in J, \gamma_{J}^{C}(m)=0 \vee \gamma_{J}^{L}(m)=0}{\bigcup_{J}^{*},} \\
& X^{S}(m) \subset X^{S}(\widehat{m}), \quad X^{S}(m)=X^{S}(\widehat{m}) \backslash \\
& \bigcup_{i \notin H, \gamma_{H}^{C}(\widehat{m})=1 \vee \gamma_{H}^{L}(\widehat{m})=2} V_{H}^{*},
\end{aligned}
$$

where $V_{J}^{*}$ is $V_{J}^{C}$ if $\gamma_{J}^{C}(m)=0$ or $V_{J}^{L}$ if $\gamma_{J}^{L}(m)=0$ and in the same way $V_{H}^{*}$ is $V_{H}^{C}$ if $\gamma_{H}^{C}(\widehat{m})=1$ or $V_{H}^{L}$ if $\gamma_{H}^{L}(\widehat{m})=2$.

At the end, we can illustrate the inclusions of the open sets of stable and semi-stable points, with the following diagrams:

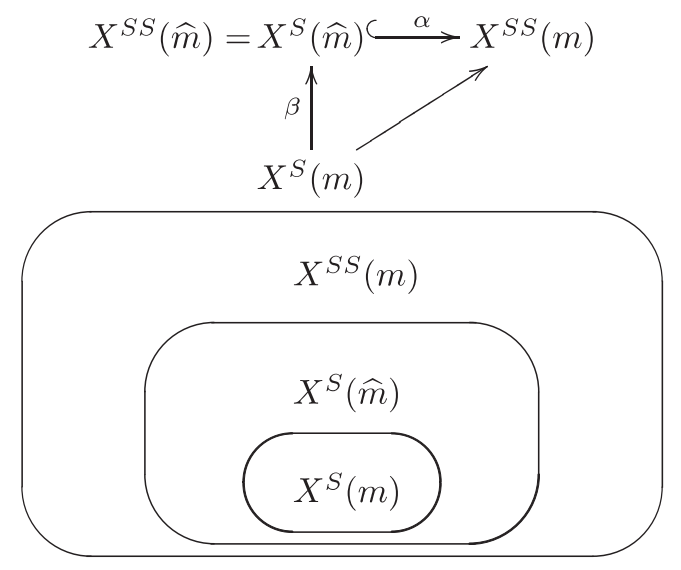

The inclusions $X^{S}(m) \subset X^{S}(\widehat{m}) \subset X^{S S}(m)$ induce a morphism

$$
\theta: X^{S}(\widehat{m}) / G \longrightarrow X^{S S}(m) / / G
$$

which is an isomorphism over $X^{S}(m) / G$, while over $\left(X^{S S}(m) / / G\right) \backslash$ $\backslash\left(X^{S}(m) / G\right)$ it is a contraction of subvarieties. In fact, consider a point $\xi \in\left(X^{S S}(m) / / G\right) \backslash\left(X^{S}(m) / G\right)$ : this is the image in $X^{S S}(m) / / G$ of different, strictly semi-stable orbits, that all have in their closure a closed, minimal orbit $G x$, for a certain configuration $x=\left(x_{1}, \ldots, x_{n}\right) \in X^{S S S}(m)$. In particular this configuration $x$ has $|J|$ coincident points, and the others $n-|J|$ collinear; by the numerical criterion, we get $\gamma_{J}^{C}(m)=0$ and $\gamma_{J^{\prime}}^{L}(m)=0$, where $J$ indicates the coincident points, while $J^{\prime}=\{1, \ldots, n\} \backslash J$ indicates the collinear ones. 
If there are no further coincidences, we can assume $x$ has the form

$$
\left(\begin{array}{ccccccccc}
1 & \ldots & 1 & 0 & 0 & 0 & 0 & \ldots & 0 \\
0 & \ldots & 0 & 1 & 0 & 1 & 1 & \ldots & 1 \\
0 & \ldots & 0 & 0 & 1 & \beta_{1} & \beta_{2} & \ldots & \beta_{n-|J|-2}
\end{array}\right), \beta_{k} \in \mathbb{C}^{*}, \forall k .
$$

The orbits $O$ that contain $G x$ in their closure, are characterized by $\gamma_{J}^{C}(m)=0$ or $\gamma_{J^{\prime}}^{L}(m)=0$; there are two different cases:

1. $\gamma_{J}^{C}(m)=0$ : orbits look like

$$
O_{1}=G \cdot\left(\begin{array}{ccccccccc}
1 & \ldots & 1 & 0 & 0 & \alpha_{1} & \alpha_{2} & \ldots & \alpha_{n-|J|-2} \\
0 & \ldots & 0 & 1 & 0 & 1 & 1 & \ldots & 1 \\
0 & \ldots & 0 & 0 & 1 & \rho \beta_{1} & \rho \beta_{2} & \ldots & \rho \beta_{n-|J|-2}
\end{array}\right), \rho \in \mathrm{C}^{*}, \alpha_{k} \in \mathrm{C} .
$$

2. $\gamma_{J^{\prime}}^{L}(m)=0$ : orbits look like

$$
O_{2}=G \cdot\left(\begin{array}{cccccccccc}
1 & 1 & \ldots & 1 & 0 & 0 & 0 & 0 & \ldots & 0 \\
0 & \delta_{1} & \ldots & \delta_{|J|-1} & 1 & 0 & 1 & 1 & \ldots & 1 \\
0 & \varepsilon_{1} & \ldots & \varepsilon_{|J|-1} & 0 & 1 & \beta_{1} & \beta_{2} & \ldots & \beta_{n-|J|-2}
\end{array}\right), \delta_{k}, \varepsilon_{k} \in \mathrm{C} .
$$

Now, calculating $\theta^{-1}(\xi)$, it follows that:

$$
\theta^{-1}(\xi)=\theta^{-1}(\phi(x))
$$

by the numerical criterion, only one between $V_{J}^{C}$ and $V_{J^{\prime}}^{L}$ is included in $X^{S}(\widehat{m})$.

Dealing with an elementary transformation of the first type $\left(\widehat{m} \stackrel{+1_{i}}{\longrightarrow} m\right)$, then

- if $i \in J \Rightarrow \theta^{-1}(\xi)=\theta^{-1}\left(\phi\left(\overline{V_{J}^{C}} \cup \overline{V_{J^{\prime}}^{L}}\right)\right)=\widehat{\phi}$ (orbits of type $\left.O_{1}\right)$.

When $n \geq 5$, this has dimension:

$$
d=n-|J|-3 .
$$

In fact, let us consider the minimal closed orbit $G x$ : all the orbits that contain $G x$ in their closure and are stable in $X^{S}(\widehat{m})$, are characterized by the coincidence of $|J|$ points $\left(O_{1}\right.$ orbits).

- if $i \in J^{\prime} \Rightarrow \theta^{-1}(\xi)=\theta^{-1}\left(\phi\left(\overline{V_{J}^{C}} \cup \overline{V_{J^{\prime}}^{L}}\right)\right)=\widehat{\phi}$ (orbits of type $O_{2}$ ).

Now the dimension $d$ of $\theta^{-1}(\xi)$ is

$$
d=2\left(n-\left|J^{\prime}\right|-1\right)-1 .
$$

Dealing with an elementary transformation of the second type $\left(\widehat{m} \stackrel{-1_{i}}{\longrightarrow} m\right)$, then

$$
i \in J \Rightarrow d=2\left(n-\left|J^{\prime}\right|-1\right)-1 ; \quad i \in J^{\prime} \Rightarrow d=n-|J|-3 .
$$


2. $X=\mathbb{P}^{2}(\mathrm{C})^{5}$.

2.1 - Number of quotients.

Let us study the case $n=5: X=\mathbb{P}^{2}(\mathbb{C})^{5}$. Let $m=\left(m_{1}, \ldots, m_{5}\right)$ be a polarization such that

$$
0<m_{i}<\frac{1}{3}, \quad m_{i} \geq m_{i+1}, \quad|m|=1 .
$$

After normalization of $|m|$ and possible permutation of the factors, this is equivalent by Proposition 1.5 to assuming that $X^{S}(m) \neq \emptyset$. It is easy to see that $x \in X$ is unstable (that is, not semi-stable) if any of the following holds

- three of the $x_{i}$ are coincident;

- four of the $x_{i}$ are collinear;

- there are two coincident pairs of $x_{i}$;

- any of the pairs $x_{i}, x_{j}$ with $i j=12,13,14,23,24$ are coincident.

In fact, if any of these possibilities satisfies the semi-stability condition, there exists $k$ with $m_{k} \geq \frac{1}{3}$, contradicting (8). It follows that the following sets are always included in $X^{S}(m)$ :

$$
V_{135}^{L}, \quad V_{145}^{L}, \quad V_{235}^{L}, \quad V_{245}^{L}, \quad V_{345}^{L},
$$

while the following sets may or may not be included in $X^{S}(m)$ :

$$
\begin{array}{ccccc}
V_{15}^{C}, & V_{25}^{C}, & V_{34}^{C}, & V_{35}^{C}, & V_{45}^{C}, \\
V_{234}^{L}, & V_{134}^{L}, & V_{125}^{L}, & V_{124}^{L}, & V_{123}^{L} .
\end{array}
$$

In view of the excluded sets listed above and Remark 1.4, these are the only sets we need to consider in order to determine $X^{S}(m)$ and $X^{S S}(m)$. Moreover, the sets in (10) pair off in an obvious way and, for each pair, either one member of the pair is contained in $X^{S}(m)$ and the other member is contained in $X^{U S}(m)$ or both members are contained in $X^{S S S}(m)$.

We consider first the case in which $X^{S}(m)=X^{S S}(m)$, so that $X^{S S S}(m)=\emptyset$ : then there are precisely six different possibilities and we will show that there are exactly six different Geometric Quotients. In fact

0 . in $\mathcal{U}^{S}(m)$ there may be only sets $V_{J}^{L}$ : an example is the polarization $m=(1 / 5,1 / 5,1 / 5,1 / 5,1 / 5)$;

1. if in $\mathcal{U}^{S}(m)$ there is one set $V_{K}^{C}$, it is $V_{45}^{C}$ : in fact, for $i \neq j$, we have $m_{i}+m_{j} \geq m_{4}+m_{5}$, so $\gamma_{i j}^{C}(m)>0 \Rightarrow \gamma_{45}^{C}(m)>0$.

Example: $m=(1 / 4,1 / 4,1 / 4,1 / 8,1 / 8)$; 
2. if in $\mathcal{U}^{S}(m)$ there are two sets $V_{K}^{C}$, they are $V_{45}^{C}$ and $V_{35}^{C}$ : the argument is similar to the previous one.

Example: $m=(3 / 11,3 / 11,2 / 11,2 / 11,1 / 11)$;

3. if in $\mathcal{U}^{S}(m)$ there are three sets $V_{K}^{C}$, we can have two cases:

(a) $V_{45}^{C}, V_{35}^{C}$ and $V_{25}^{C}$, example $m=(3 / 10,1 / 5,1 / 5,1 / 5,1 / 10)$;

(b) $V_{45}^{C}, V_{35}^{C}$ and $V_{34}^{C}$, example $m=(3 / 10,3 / 10,1 / 5,1 / 10,1 / 10)$.

4. if in $\mathcal{U}^{S}(m)$ there are four sets $V_{K}^{C}$, they are $V_{45}^{C}, V_{35}^{C}, V_{25}^{C}$ and $V_{15}^{C}$. Example: $m=(1 / 4,1 / 4,1 / 4,2 / 9,1 / 36)$;

5. the case of all $V_{K}^{C}$ sets in $\mathcal{U}^{S}(m)$ is impossible, because $V_{45}^{C}, V_{35}^{C}, V_{34}^{C}$, $V_{25}^{C}$ are incompatible.

We have found six cases:

0. $\quad \mathcal{U}^{S}(m) \supseteq \bigcup\left\{V_{234}^{L}, V_{134}^{L}, V_{124}^{L}, V_{123}^{L}, V_{125}^{L}, V_{135}^{L}, V_{145}^{L}, V_{235}^{L}, V_{245}^{L}, V_{345}^{L}\right\}$

1. $\mathcal{U}^{S}(m) \supseteq \bigcup\left\{V_{234}^{L}, V_{134}^{L}, V_{124}^{L}, V_{125}^{L}, V_{45}^{C}, V_{135}^{L}, V_{145}^{L}, V_{235}^{L}, V_{245}^{L}, V_{345}^{L}\right\}$,

2. $\mathcal{U}^{S}(m) \supseteq \bigcup\left\{V_{234}^{L}, V_{134}^{L}, V_{125}^{L}, V_{35}^{C}, V_{45}^{C}, V_{135}^{L}, V_{145}^{L}, V_{235}^{L}, V_{245}^{L}, V_{345}^{L}\right\}$,

3a. $\mathcal{U}^{S}(m) \supseteq \bigcup\left\{V_{234}^{L}, V_{125}^{L}, V_{25}^{C}, V_{35}^{C}, V_{45}^{C}, V_{135}^{L}, V_{145}^{L}, V_{235}^{L}, V_{245}^{L}, V_{345}^{L}\right\}$,

$3 b . \mathcal{U}^{S}(m) \supseteq \bigcup\left\{V_{234}^{L}, V_{134}^{L}, V_{34}^{C}, V_{35}^{C}, V_{45}^{C}, V_{135}^{L}, V_{145}^{L}, V_{235}^{L}, V_{245}^{L}, V_{345}^{L}\right\}$,

4. $\mathcal{U}^{S}(m) \supseteq \bigcup\left\{V_{125}^{L}, V_{15}^{C}, V_{25}^{C}, V_{35}^{C}, V_{45}^{C}, V_{135}^{L}, V_{145}^{L}, V_{235}^{L}, V_{245}^{L}, V_{345}^{L}\right\}$.

Then there are only six different open sets of stable points and thus six geometric quotients.

Now suppose $X^{S}(m) \neq X^{S S}(m)$. Then one or more of the pairs in (10) is contained in $X^{S S S}(m)$. For such a pair, there are two distinct types of strictly semi-stable orbit:

- an orbit $O_{1}$ with $x_{k_{1}}=x_{k_{2}}, K=\left\{k_{1}, k_{2}\right\}: O_{1}=V_{K}^{C}$;

- orbits $O_{2}$ with $x_{i_{1}}, x_{i_{2}}, x_{i_{3}}$ collinear, $i_{1}, i_{2}, i_{3} \in K^{\prime}$.

Orbit $O_{1}$ and all orbits $O_{2}$ contain in their closure a closed, minimal, strictly semi-stable orbit $O_{12}$, that is characterized by $x_{k_{1}}=x_{k_{2}}$ and $x_{i_{1}}, x_{i_{2}}, x_{i_{3}}$ collinear:

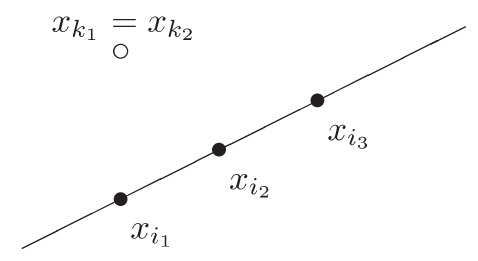


In the categorical quotient $X^{S S}(m) / / G$, orbits $O_{1}$ and $O_{2}$ determine the same point; in fact $\mathrm{O}_{12} \subset\left(\overline{\mathrm{O}_{1}} \cap \overline{\mathrm{O}_{2}}\right)$.

Let us examine the stable case more accurately: we know that only one between $O_{1}$ and $O_{2}$ is included in $X^{S}(m)$; when $O_{1}$ is included, it determines a point of the geometric quotient. In fact if for example $V_{45}^{C} \subset X^{S}(m)$, then $\phi\left(V_{45}^{C}\right) \cong\left(\mathbb{P}^{2}(\mathbb{C})^{4}\right)^{S}\left(m_{1}, m_{2}, m_{3}, m_{4}+m_{5}\right) / S L_{3}(\mathbb{C})$ which is a point (see Remark 1.7). When orbits $O_{2}$ are included in $X^{S}(m)$, they determine a $\mathrm{P}^{1}(\mathrm{C})$ in $X^{S}(m) / G$. In fact if for example $V_{123}^{L} \subset X^{S}(m)$, then we can assume

$$
O_{2}=G \cdot\left(\begin{array}{lllll}
1 & 0 & 1 & 0 & \alpha \\
0 & 1 & 1 & 0 & \beta \\
0 & 0 & 0 & 1 & 1
\end{array}\right),(\alpha, \beta) \in \mathbb{C}^{2} \backslash\{(0,0)\}
$$

Applying to $\mathrm{O}_{2}$ a projectivity $G_{\lambda}$ of $\mathrm{P}^{2}(\mathrm{C})$ that fixes the line that contains $x_{1}, x_{2}, x_{3}\left(G_{\lambda} \cong \operatorname{diag}\left(\lambda, \lambda, \lambda^{-2}\right)\right.$, with $\left.\lambda \in \mathbb{C}^{*}\right)$, it follows that

$$
G_{\lambda} \cdot x \ni\left(\begin{array}{ccccc}
1 & 0 & 1 & 0 & \lambda^{3} \alpha \\
0 & 1 & 1 & 0 & \lambda^{3} \beta \\
0 & 0 & 0 & 1 & 1
\end{array}\right)
$$

If $\alpha \neq 0$, then we can assume $\lambda^{3}=\alpha^{-1}$; thus we obtain $x_{5}=\left[1: \alpha^{-1} \beta: 1\right]$; in the same way if $\beta \neq 0$, then $x_{5}=\left[\alpha \beta^{-1}: 1: 1\right]$.

Then it is clear that $\phi\left(O_{2}\right) \cong \mathbb{P}^{1}(\mathrm{C})$.

In the semi-stable case when $V_{K}^{C}, V_{K^{\prime}}^{L} \subset X^{S S}(m)$, we know from the above that $\overline{V_{K}^{C}} \cap \overline{V_{K^{\prime}}^{L}} \neq \emptyset$ is a single non-singular point of $X^{S S}(m) / / G$, just as in the stable case when $V_{K}^{C}$ is included in the open set of stable points.

In this way it follows that every categorical quotient $X^{S S}(m) / / G$, where

$$
X^{S S}(m) \supseteq U^{\mathrm{GEN}} \cup\{\underbrace{V_{J}^{C}, V_{I}^{L}, \ldots,}_{\text {stable sets }} \underbrace{V_{K}^{C}, V_{K^{\prime}}^{L}, \ldots, V_{H}^{C}, V_{H^{\prime}}^{L}}_{\text {semi-stable sets }}\},
$$

is isomorphic to a geometric one $X^{S}\left(m^{\prime}\right) / G$, where

$$
X^{S}\left(m^{\prime}\right) \supseteq U^{\mathrm{GEN}} \cup\left\{V_{J}^{C}, V_{I}^{L}, \ldots, V_{K}^{C}, \cdots, V_{H}^{C}\right\} .
$$

The polarization $m^{\prime}$ is obtained from $m$ using elementary transformations such that for each $\overline{V_{K}^{C}} \cap \overline{V_{K^{\prime}}^{L}} \neq \emptyset$ in $X^{S S_{(}}(m)$, then $V_{K}^{C} \subset X^{S}\left(m^{\prime}\right)$. This is always possible because the number of different quotients is finite.

THEOREM 2.8. Let $X=\mathbb{P}^{2}(\mathbb{C})^{5}$ : then there are six non trivial quotients.

Moreover a quotient $X^{S S}(m) / / G$ is isomorphic to one of the following: 


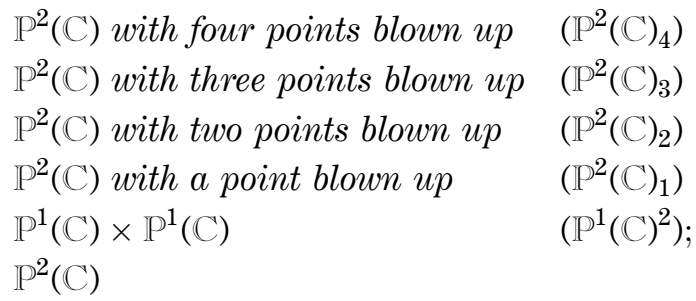

Proof. The six different open sets of stable points (11) correspond to six different quotients:

0. $X^{S}(m) / G \cong \mathbb{P}^{2}(\mathrm{C})$ with four points blown up

1. $X^{S}(m) / G \cong \mathbb{P}^{2}(\mathrm{C})$ with three points blown up

2. $X^{S}(m) / G \cong \mathbb{P}^{2}(\mathrm{C})$ with two points blown up

3a. $X^{S}(m) / G \cong \mathbb{P}^{2}(C)$ with a point blown-up

3b. $X^{S}(m) / G \cong \mathbb{P}^{1}(\mathrm{C}) \times \mathbb{P}^{1}(\mathrm{C})$

4. $X^{S}(m) / G \cong \mathbb{P}^{2}(\mathrm{C})$

The proof examines the different cases pointed out in the description of the open set of stable points $X^{S}(m)$ :

$$
X^{S}(m)=U^{\mathrm{GEN}} \cup \mathcal{U}^{S}(m) .
$$

Case 4.

$$
X^{S}(m) \supseteq U^{\mathrm{GEN}} \cup\left\{V_{125}^{L}, V_{15}^{C}, V_{25}^{C}, V_{35}^{C}, V_{45}^{C}, V_{135}^{L}, V_{145}^{L}, V_{235}^{L}, V_{245}^{L}, V_{345}^{L}\right\}
$$

Stable configurations have $x_{1} x_{2} x_{3} x_{4}$ in general position, while $x_{5}$ is free in $\mathbb{P}^{2}(\mathbb{C})$ (in particular it may be coincident with the other points).

Then

$$
X^{S}(m) / G \cong \mathbb{P}^{2}(\mathbb{C})
$$

Case 3a.

$$
X^{S}(m) \supseteq U^{\mathrm{GEN}} \cup\left\{V_{125}^{L}, V_{234}^{L}, V_{25}^{C}, V_{35}^{C}, V_{45}^{C}, V_{135}^{L}, V_{145}^{L}, V_{235}^{L}, V_{245}^{L}, V_{345}^{L}\right\}
$$

There are two different kinds of stable configurations:

- $x_{1} x_{2} x_{3} x_{4}$ in general position, $x_{5}$ cannot be coincident with $x_{1}$ (i.e. applying the projectivity of $\mathbb{P}^{2}(\mathrm{C})$ that sends $x_{1}, x_{2}, x_{3}, x_{4}$ to $[1: 0: 0],[0: 1: 0],[0: 0: 1],[1: 1: 1]$, then $\left.x_{5} \in \mathbb{P}^{2}(\mathbb{C}) \backslash\{[1: 0: 0]\}\right)$;

- $x_{2} x_{3} x_{4}$ collinear ("complementary" condition of $x_{5}=x_{1}$ ); using a projectivity of $\mathbb{P}^{2}(\mathrm{C})$ we obtain: 


$$
\left(\begin{array}{lllll}
1 & 0 & 0 & 0 & 1 \\
0 & 1 & 0 & 1 & \alpha \\
0 & 0 & 1 & 1 & \beta
\end{array}\right), \quad(\alpha, \beta) \in \mathbb{C}^{2} \backslash\{(0,0)\}
$$

Then with another projectivity $G_{\lambda}$ of $\mathrm{P}^{2}(\mathrm{C})$, that fixes the line containing $x_{2} x_{3} x_{4}$, we get: $\left(G_{\lambda}=\operatorname{diag}\left(\lambda^{-2}, \lambda, \lambda\right), \lambda \in \mathbb{C}^{*}\right)$

$$
\left(\begin{array}{ccccc}
1 & 0 & 0 & 0 & 1 \\
0 & 1 & 0 & 1 & \lambda^{3} \alpha \\
0 & 0 & 1 & 1 & \lambda^{3} \beta
\end{array}\right)
$$

If $\alpha \neq 0$, then assuming $\lambda^{3}=\alpha^{-1}$, we obtain $x_{5}=\left[1: 1: \alpha^{-1} \beta\right]$; in the same way if $\beta \neq 0$, taking $\lambda^{3}=\beta^{-1}$, we get $x_{5}=\left[1: \alpha \beta^{-1}: 1\right]$. Passing to the quotient we get a cover of $\mathrm{P}^{1}(\mathrm{C})$.

Comparing this case to the previous one, in $X^{S}(m)$ the set $U_{15}^{C}$ (that determines a point of the quotient) is substituted by $U_{234}^{L}$ that gives $\mathbb{P}^{1}(\mathbb{C})$ in the quotient. Then

$$
X^{S}(m) / G \cong \mathbb{P}^{2}(\mathbb{C}) \text { with a blow-up } .
$$

Case $3 \mathrm{~b}$. In this case, if $x_{1}, x_{2}, x_{3}, x_{4}$ are in general position, $x_{5}$ cannot be collinear with $x_{1}, x_{2}$. As in the previous case, the equality $x_{1}=x_{5}$ is replaced by the collinearity of $x_{2}, x_{3}, x_{4}$, giving rise to a blowingup of the corresponding point of $\mathrm{P}^{2}(\mathbb{C})$. The same applies to the equality $x_{2}=x_{5}$. The proper transform of the line joining $x_{1}, x_{2}$ corresponds to the equality $x_{3}=x_{4}$, which is allowed, so we must blow down this line to obtain $\mathbb{P}^{1}(\mathbb{C}) \times \mathbb{P}^{1}(\mathbb{C})$.

The other cases are analogous to the first two.

$2.2-$ Quotients $\mathrm{P}^{2}(\mathrm{C})^{5} / / G$.

The following diagram shows some birational maps between quotients (the polarization is given in brackets with the corresponding quotient as a subscript); for example if $m=(22211)$, then $X^{S}(m)=\mathbb{P}^{2}(\mathbb{C})_{3}$ (i.e. $\mathbb{P}^{2}(\mathbb{C})$ with three points blown-up) and there is a morphism

$\theta: X^{S}(22211) / G=X^{S}(44422) / G=\mathbb{P}^{2}(\mathbb{C})_{3} \rightarrow X^{S S}(44322) / / G=\mathbb{P}^{1}(\mathbb{C}) \times \mathbb{P}^{1}(\mathbb{C})$

In fact $\widehat{m}=(44422)$ is an elementary transformation of $m=(44322)$ in the sense of Section 1.3 and $\theta$ is the map given by (4). 
The diagram has been obtained by direct calculation (some of the techniques will be illustrated in Section 3); in particular it includes some cases where $X^{S}(m)=\emptyset$ which are not included in the earlier discussion and where the quotient has dimension smaller than two. For example: $X(21111) \cong\left(\mathbb{P}^{1}(\mathrm{C})\right)^{4}(1111)$ that determines a one-dimensional categorical quotient, while $X(33111) \cong \mathbb{P}^{0}(\mathrm{C})$ that determines a zero-dimensional categorical quotient (compare the proof of Proposition 1.5)

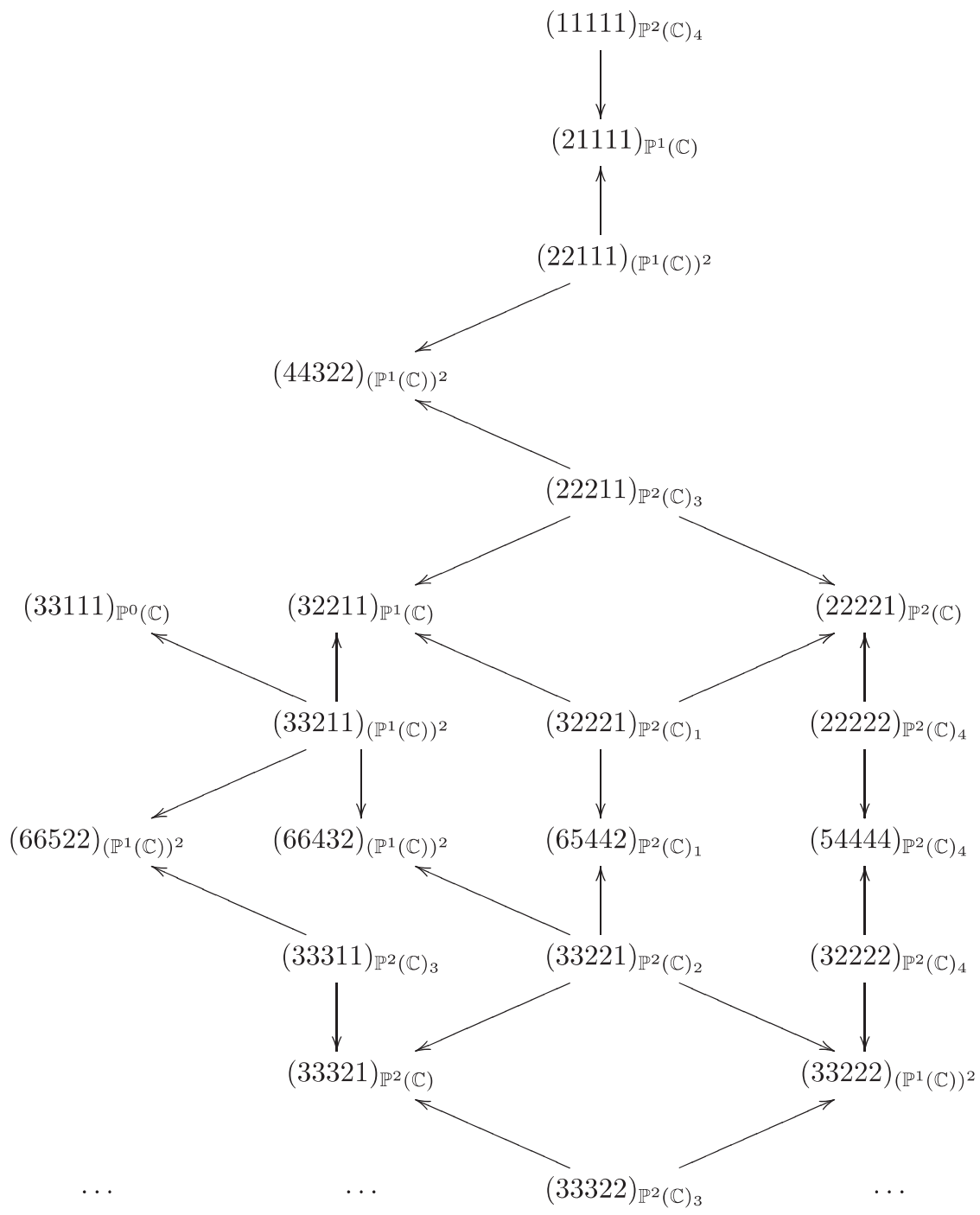


3. $X=\mathbb{P}^{2}(\mathrm{C})^{6}$.

\section{1 - Number of quotients.}

Now we study the case $n=6: X=\mathbb{P}^{2}(\mathrm{C})^{6}$; as in the previous case we first determine how many different quotients we can get when $X^{S}(m)=X^{S S}(m)$ and the polarization varies.

For a polarization $m=\left(m_{1}, \ldots, m_{6}\right)$ such that $X^{S}(m) \neq \emptyset$ and $X^{S}(m)=X^{S S}(m)$, then

$$
X^{S}(m)=U^{\mathrm{GEN}} \cup \mathcal{U}^{S}(m) .
$$

We want to describe the structure of the sets $\mathcal{U}^{S}(m)$; assume that $0<m_{i}<\frac{1}{3}, m_{i} \geq m_{i+1},|m|=1$.

We are interested in those sets $V_{K_{1}, \ldots, K_{r}}^{C}$ that are included in $X^{S}(m)$ : some are always included in $X^{S}(m)$ :

$$
V_{36}^{C}, \quad V_{46}^{C}, \quad V_{56}^{C},
$$

and others may be included in $X^{S}(m)$ : the general sets $V_{K}^{C}$

$$
\begin{array}{lllllllll}
V_{15}^{C}, & V_{16}^{C}, & V_{23}^{C}, & V_{24}^{C}, & V_{25}^{C}, & V_{26}^{C}, & V_{34}^{C}, & V_{35}^{C}, & V_{45}^{C}, \\
V_{156}^{C}, & V_{256}^{C}, & V_{345}^{C}, & V_{346}^{C}, & V_{356}^{C}, & V_{456}^{C}, & & &
\end{array}
$$

and also their "combinations" $V_{K_{1}, K_{2}}^{C}$, with $\left|K_{1}\right|=\left|K_{2}\right|=2$, disjoint subsets of $\{1, \ldots, 6\}$. As we shall see the number of different sets $\mathcal{U}^{S}(m)$ is 38 : so the number of chambers in which the $G$-ample cone of Dolgachev and $\mathrm{Hu}$ [4] is divided is less than or equal to 38 .

First of all the minimum number of general sets $V_{K}^{C}$ with $|K|=2$, included in $X^{S}(m)$ is five: in fact for example consider only the sets $V_{36}^{C}, V_{46}^{C}, V_{56}^{C}$ that are always included in $X^{S}(m)$, then obviously

$$
m_{1}+m_{6}>\frac{1}{3}, m_{2}+m_{5}>\frac{1}{3}, m_{3}+m_{4}>\frac{1}{3} \Rightarrow \sum_{i=1}^{6} m_{i}>1: \text { impossible. }
$$

In a similar way it is impossible to have only four sets $V_{K}^{C}(|K|=2)$ in $X^{S}(m)$.

Then for five sets $V_{K}^{C}$, we have $V_{16}^{C}, V_{26}^{C}, V_{36}^{C}, V_{46}^{C}, V_{56}^{C}$ : in fact with another 5-tuple (for example $V_{45}^{C}, V_{26}^{C}, V_{36}^{C}, V_{46}^{C}, V_{56}^{C}$ ), we have $|m|>1$, which is impossible. Moreover with these combinations, it is impossible to obtain a set as $V_{K}^{C}$ with $|K|=3$.

Going on with the calculations, we are able to construct the following table, 
TABle 3.1.

\begin{tabular}{|c|c|c|c|c|c|}
\hline $\begin{array}{c}V_{K}^{C} \\
|K|=2\end{array}$ & $\begin{array}{l}\text { No } V_{K}^{C} \\
|K|=3\end{array}$ & $\begin{array}{c}1 \text { set } V_{K}^{C}, \\
|K|=3\end{array}$ & $\begin{array}{c}2 \text { sets } V_{K}^{C}, \\
|K|=3\end{array}$ & $\begin{array}{c}3 \text { sets } V_{K}^{C}, \\
|K|=3\end{array}$ & $\begin{array}{l}4 \text { sets } V_{K}^{C} \\
\quad|K|=3\end{array}$ \\
\hline $\begin{array}{c}V_{16}^{C}, V_{26}^{C}, V_{36}^{C} \\
V_{46}^{C}, V_{56}^{C}\end{array}$ & $\begin{array}{c}\checkmark \\
\frac{1}{11}(222221)\end{array}$ & $\mathrm{No}^{(*)}$ & No & No & No \\
\hline $\begin{array}{l}V_{16}^{C}, V_{26}^{C}, V_{36}^{C} \\
V_{45}^{C}, V_{46}^{C}, V_{56}^{C}\end{array}$ & $\begin{array}{c}\checkmark \\
\frac{1}{14}(333221)\end{array}$ & $\begin{array}{c}V_{456}^{C} \\
\frac{1}{17}(444221)\end{array}$ & $\mathrm{No}^{(*)}$ & No & No \\
\hline $\begin{array}{l}V_{34}^{C}, V_{35}^{C}, V_{36}^{C}, \\
V_{45}^{C}, V_{46}^{C}, V_{56}^{C}\end{array}$ & $\begin{array}{c}\checkmark \\
\frac{1}{8}(221111)\end{array}$ & $\begin{array}{c}V_{456}^{C} \\
\frac{1}{11}(332111)\end{array}$ & $\begin{array}{c}V_{456}^{C}, V_{356}^{C} \\
\frac{1}{14}(442211)\end{array}$ & $\begin{array}{c}V_{456}^{C}, V_{356}^{C}, \\
V_{346}^{C} \\
\frac{1}{17}(552221)\end{array}$ & $\begin{array}{c}V_{456}^{C}, V_{356}^{C}, \\
V_{346}^{C}, V_{345}^{C} \\
\frac{1}{10}(331111)\end{array}$ \\
\hline $\begin{array}{c}V_{25}^{C}, V_{26}^{C}, V_{35}^{C}, \\
V_{36}^{C}, V_{45}^{C}, V_{46}^{C}, \\
V_{56}^{C}\end{array}$ & $\begin{array}{c}\checkmark \\
\frac{1}{11}(322211)\end{array}$ & $\begin{array}{c}V_{456}^{C} \\
\frac{1}{14}(433211)\end{array}$ & $\begin{array}{c}V_{456}^{C}, V_{356}^{C} \\
\frac{1}{17}(543311)\end{array}$ & $\begin{array}{c}V_{456}^{C}, V_{356}^{C}, \\
V_{256}^{C} \\
\frac{1}{19}(644311)\end{array}$ & $\mathrm{No}^{(*)}$ \\
\hline $\begin{array}{c}V_{26}^{C}, V_{34}^{C}, V_{35}^{C}, \\
V_{36}^{C}, V_{45}^{C}, V_{46}^{C}, \\
V_{56}^{C}\end{array}$ & $\begin{array}{c}\checkmark \\
\frac{1}{14}(432221)\end{array}$ & $\begin{array}{c}V_{456}^{C} \\
\frac{1}{17}(543221)\end{array}$ & $\begin{array}{c}V_{456}^{C}, V_{356}^{C} \\
\frac{1}{26}(875321)\end{array}$ & $\begin{array}{l}V_{456}^{C}, V_{356}^{C}, \\
\frac{1}{16}(542221)\end{array}$ & $\mathrm{No}^{(* *)}$ \\
\hline $\begin{array}{c}V_{16}^{C}, V_{26}^{C}, V_{35}^{C}, \\
V_{36}^{C}, V_{45}^{C}, V_{46}^{C} \\
V_{56}^{C}\end{array}$ & $\begin{array}{c}\checkmark \\
\frac{1}{17}(443321)\end{array}$ & $\begin{array}{c}V_{456}^{C} \\
\frac{1}{20}(554321)\end{array}$ & $\begin{array}{c}V_{456}^{C}, V_{356}^{C} \\
\frac{1}{26}(775421)\end{array}$ & $\mathrm{No}^{(*)}$ & No \\
\hline $\begin{array}{c}V_{16}^{C}, V_{26}^{C}, V_{34}^{C}, \\
V_{35}^{C}, V_{36}^{C}, V_{45}^{C}, \\
V_{46}^{C}, V_{56}^{C}\end{array}$ & $\begin{array}{c}\checkmark \\
\frac{1}{13}(332221)\end{array}$ & $\begin{array}{c}V_{456}^{C} \\
\frac{1}{16}(443221)\end{array}$ & $\begin{array}{c}V_{456}^{C}, V_{356}^{C} \\
\frac{1}{19}(553321)\end{array}$ & $\begin{array}{c}V_{456}^{C}, V_{356}^{C}, \\
V_{346}^{C} \\
\frac{1}{25}(774331)\end{array}$ & $\mathrm{No}^{(* *)}$ \\
\hline $\begin{array}{c}V_{16}^{C}, V_{25}^{C}, V_{26}^{C}, \\
V_{35}^{C}, V_{36}^{C}, V_{45}^{C}, \\
V_{46}^{C}, V_{56}^{C}\end{array}$ & $\frac{1}{16}(433321)$ & $\begin{array}{c}V_{456}^{C} \\
\frac{1}{26}(766421)\end{array}$ & $\begin{array}{c}V_{456}^{C}, V_{356}^{C} \\
\frac{1}{26}(765521)\end{array}$ & $\begin{array}{c}V_{456}^{C}, V_{356}^{C}, \\
V_{256}^{C} \\
\frac{1}{25}(755521)\end{array}$ & $\mathrm{No}^{(*)}$ \\
\hline $\begin{array}{c}V_{25}^{C}, V_{26}^{C}, V_{34}^{C}, \\
V_{35}^{C}, V_{36}^{C}, V_{45}^{C}, \\
V_{46}^{C}, V_{56}^{C}\end{array}$ & $\begin{array}{c}\checkmark \\
\frac{1}{31}(965542)\end{array}$ & $\begin{array}{c}V_{456}^{C} \\
\frac{1}{26}(865322)\end{array}$ & $\begin{array}{c}V_{456}^{C}, V_{356}^{C} \\
\frac{1}{13}(432211)\end{array}$ & $\mathrm{No}^{(\dagger)}$ & No \\
\hline
\end{tabular}


TABLE 3.1. Segue.

\begin{tabular}{|c|c|c|c|c|c|}
\hline $\begin{array}{c}V_{K}^{C}, \\
|K|=2\end{array}$ & $\begin{array}{l}\text { No } V_{K}^{C} \\
|K|=3\end{array}$ & $\begin{array}{c}1 \text { set } V_{K}^{C} \text {, } \\
|K|=3\end{array}$ & $\begin{array}{c}2 \text { sets } V_{K}^{C} \\
\quad|K|=3\end{array}$ & $\begin{array}{c}3 \text { sets } V_{K}^{C} \\
|K|=3\end{array}$ & $\begin{array}{c}4 \text { sets } V_{K}^{C} \\
|K|=3\end{array}$ \\
\hline $\begin{array}{l}V_{15}^{C}, V_{16}^{C}, V_{25}^{C}, \\
V_{26}^{C}, V_{35}^{C}, V_{36}^{C}, \\
V_{45}^{C}, V_{46}^{C}, V_{56}^{C}\end{array}$ & $\begin{array}{c}\checkmark \\
\frac{1}{10}(222211)\end{array}$ & $\begin{array}{c}V_{456}^{C} \\
\frac{1}{13}(333211)\end{array}$ & $\begin{array}{c}V_{456}^{C}, V_{356}^{C} \\
\frac{1}{16}(443311)\end{array}$ & $\begin{array}{c}V_{456}^{C}, V_{356}^{C}, \\
V_{256}^{C} \\
\frac{1}{25}(766411)\end{array}$ & $\begin{array}{c}V_{456}^{C}, V_{356}^{C}, \\
V_{256}^{C}, V_{156}^{C} \\
\frac{1}{22}(555511)\end{array}$ \\
\hline $\begin{array}{l}V_{24}^{C}, V_{25}^{C}, V_{26}^{C}, \\
V_{34}^{C}, V_{35}^{C}, V_{36}^{C}, \\
V_{45}^{C}, V_{46}^{C}, V_{56}^{C}\end{array}$ & 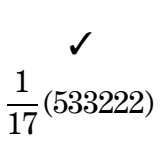 & $\begin{array}{c}V_{456}^{C} \\
\frac{1}{10}(322111)\end{array}$ & $\mathrm{No}^{(\dagger \dagger)}$ & No & No \\
\hline $\begin{array}{c}V_{23}^{C}, V_{24}^{C}, V_{25}^{C}, \\
V_{26}^{C}, V_{34}^{C}, V_{35}^{C}, \\
V_{36}^{C}, V_{45}^{C}, V_{46}^{C}, \\
V_{56}^{C}\end{array}$ & $\begin{array}{c}\checkmark \\
\frac{1}{7}(211111)\end{array}$ & $\mathrm{No}^{(\dagger \dagger \dagger)}$ & No & No & No \\
\hline
\end{tabular}

$\left(^{*}\right)$ This case is not possible, because there is not any available term;

$\left(^{* *}\right) V_{345}^{C}$ is not included in $X^{S}(m)$, because otherwise $m_{3}+m_{4}+m_{5}<\frac{1}{3}$, $m_{2}+m_{6}<\frac{1}{3} \Rightarrow m_{1}>\frac{1}{3}$, which is impossible;

(') $V_{256}^{C}, V_{345}^{C}, V_{346}^{C} \nsubseteq X^{S}(m)$;

(†) $V_{246}^{C}, V_{256}^{C}, V_{345}^{C}, V_{346}^{C}, V_{356}^{C} \not \subset X^{S}(m)$;

(††) $V_{236}^{C}, V_{246}^{C}, V_{256}^{C}, V_{345}^{C}, V_{346}^{C}, V_{356}^{C}, V_{456}^{C} \nsubseteq X^{S}(m)$.

that shows all the possible cases (in the "admissible" cells we exhibit an example of a polarization that realizes the geometric quotient). In particular it is not possible to have more than ten sets $V_{K}^{C}(|K|=2)$ in $X^{S}(m)$ : we would obtain $|m|<1$.

\section{2 - Singularities.}

In this section we study the singularities which appear in the categorical quotients when $X^{S S S}(m) \neq \emptyset$.

We suppose always that $X^{S}(m) \neq \emptyset$, so that $m_{i}<|m| / 3$ for all $i$. Suppose that $|m|$ is divisible by 3 and that there exist strictly semi-stable orbits (included in $X^{S S S}(m)$ ); then we can have different cases depending on some "partitions" of the polarization $m \in Z_{>0}^{6}$ : 
1. there are two distinct indices $i, j$ such that $m_{i}+m_{j}=|m| / 3$; as a consequence, for the other indices we have $m_{h}+m_{k}+m_{l}+m_{n}=$ $=2|m| / 3$.

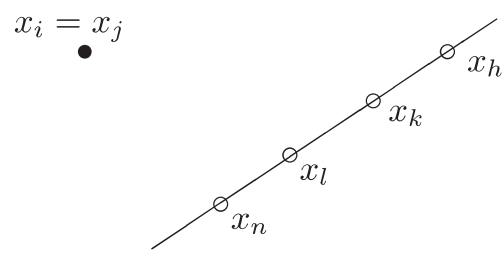

In $X^{S S}(m) / / G$ these orbits determine a curve $C_{i j} \cong \mathrm{P}^{1}(\mathbb{C})$.

If there do not exist indices $h, l$, distinct from each other and from $i$ and $j$ with $m_{h}+m_{l}=|m| / 3$, then all the orbits are closed.

1.1 particular case: $m_{i}+m_{j}=m_{h}+m_{l}=m_{k}+m_{n}=|m| / 3$ for distinct indexes (i.e. there is a "special" minimal, closed orbit other than the orbits previously seen, characterized by $\left.x_{i}=x_{j}, x_{h}=x_{l}, x_{k}=x_{n}\right)$.

$$
x_{i}=x_{j}
$$

$$
x_{h}=x_{l}
$$

$$
x_{k}=x_{n}
$$

2. there are three distinct indices $h, i, j$ such that $m_{h}+m_{i}+m_{j}=|m| / 3$; as a consequence for the other indices it holds $m_{k}+m_{l}+m_{n}=2|m| / 3$ (i.e. there is a minimal, closed orbit such that $x_{h}=x_{i}=x_{j}$, and $x_{k}, x_{l}, x_{n}$ collinear and distinct for the numerical criterion).

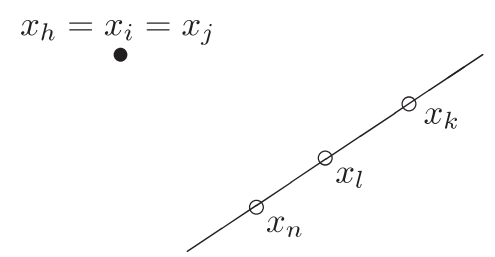

Let us study minimal, closed orbits and what they determine in $X^{S S}(m) / / G$.

3.2.1 $-x_{i}=x_{j}$ and $x_{h}, x_{k}, x_{l}, x_{n}$ collinear.

Consider a polarization $m=\left(m_{1}, \ldots, m_{6}\right)$ as previously indicated and an orbit $G x$ such that $x_{i}=x_{j}\left(m_{i}+m_{j}=|m| / 3\right)$, and the other four points $x_{h}, x_{k}, x_{l}, x_{n}$ collinear $\left(m_{h}+m_{k}+m_{l}+m_{n}=2|m| / 3\right)$. 
$G x$ is a minimal, closed, strictly semi-stable orbit and its image in $X^{S S}(m) / / G$ is a point $\xi \in C_{i j}$. For the sake of generality, suppose that $x_{h}, x_{k}, x_{l}, x_{n}$ are collinear, but distinct; for example assume $x$ as:

$$
x=\left(\begin{array}{llllll}
1 & 1 & 0 & 0 & 0 & 0 \\
0 & 0 & 1 & 0 & 1 & 1 \\
0 & 0 & 0 & 1 & a & b
\end{array}\right), \quad a, b \in \mathbb{C}^{*}, a \neq b .
$$

Now let us apply the Luna Étale Slice Theorem, to make a local study of $\xi$ : in fact it states that if $G x$ is a closed semi-stable orbit and $\xi$ is the corresponding point of $X^{S S}(m) / / G$, then the pointed varieties $\left(X^{S S}(m) / / G, \xi\right)$ and $\left(N_{x} / / G_{x}, 0\right)$ are locally isomorphic in the étale topology, where $N_{x}=N_{G x / X, x}$ is the fiber over $x$ of the normal bundle of $G x$ in $X$ (for more details about the Étale Slice Theorem, see [9], [12] and [5]).

In our case the dimension of the stabilizer $G_{x}$ is equal to one and $G_{x} \cong\left\{\operatorname{diag}\left(\lambda^{-2}, \lambda, \lambda\right), \lambda \in \mathbb{C}^{*}\right\} \cong \mathbb{C}^{*}$. Moreover the orbit $G x$ is a 7 -dimensional regular variety in $\mathrm{C}^{12}$ and the space $T_{x} \mathrm{C}^{12}=\mathrm{C}^{12}$ can be decomposed $G_{x}$-invariantly as the direct sum $T_{x} G x \oplus N_{x}$.

For a local study, first we dehomogenizate each copy of $\mathrm{P}^{2}(\mathrm{C})$, the first four copies of $\mathrm{P}^{2}(\mathrm{C})$ via the unique non-zero coordinate, the last two copies via the second coordinate; in this way $x \in \mathbb{P}^{2}(\mathbb{C})^{6}$ can be considered locally in $\mathbb{C}^{12}$ as

$$
x=\left(\begin{array}{llllll}
0 & 0 & 0 & 0 & 0 & 0 \\
0 & 0 & 0 & 0 & a & b
\end{array}\right)=(0,0,0,0,0,0,0,0,0, a, 0, b) \in \mathbb{C}^{12} .
$$

Let us consider $\mathbb{C}^{12}$ with coordinates $\left(y_{1}, \ldots, y_{12}\right)$ : the equations of the 7-dimensional tangent space $T_{x} G x$ and of the 5-dimensional normal space $N_{x}$ are

$$
T_{x} G x:\left\{\begin{array}{l}
y_{1}-y_{3}=0 \\
y_{2}-y_{4}=0 \\
y_{5}+a y_{7}-y_{9}=0 \\
y_{5}+b y_{7}-y_{11}=0 \\
(a-b) y_{6}+a b(a-b) y_{8}+b y_{10}-a y_{12}=0
\end{array} \quad N_{x}:\left\{\begin{array}{l}
y_{1}+y_{3}=0 \\
y_{2}+y_{4}=0 \\
y_{5}+y_{9}+y_{11}=0 \\
y_{7}+a y_{9}+b y_{11}=0 \\
y_{6}+y_{10}+y_{12}=0 \\
a b y_{6}-y_{8}=0 \\
a y_{10}+b y_{12}=0
\end{array}\right.\right.
$$

Then a basis for $N_{x} \cong C^{5}$ is determined by $\left\{v_{1}, \ldots, v_{5}\right\}$, where:

$$
\begin{gathered}
v_{1}=(1,0,-1,0,0,0,0,0,0,0,0,0), v_{2}=(0,1,0,-1,0,0,0,0,0,0,0,0), \\
v_{3}=(0,0,0,0,1,0, a, 0,-1,0,0,0), v_{4}=(0,0,0,0,1,0, b, 0,0,0,-1,0), \\
v_{5}=(0,0,0,0,0, a-b, 0, a b(a-b), 0, b, 0,-a) .
\end{gathered}
$$


Now, by the Étale Slice Theorem, we have to study the action of the torus $\mathrm{C}^{*}$ on $N_{x}$ : this action is induced by the diagonal action of $S L_{3}(\mathrm{C})$ on $\mathbb{P}^{2}(C)^{6}(m)$ :

$$
\begin{array}{ccccc}
\mathbb{C}^{*} & \times & \mathbb{P}^{2}(\mathbb{C}) & \rightarrow & \mathbb{P}^{2}(\mathbb{C}) \\
\left.\left(\begin{array}{ccc}
\lambda^{-2} & 0 & 0 \\
0 & \lambda & 0 \\
0 & 0 & \lambda
\end{array}\right) \quad, \quad\left[x_{i 0}: x_{i 1}: x_{i 2}\right]\right) & \mapsto \quad\left[\lambda^{-2} x_{i 0}: \lambda x_{i 1}: \lambda x_{i 2}\right]
\end{array}
$$

Then on the coordinates $\left(y_{1}, \ldots, y_{12}\right)$ of $\mathrm{C}^{12}$ we have:

$$
\begin{aligned}
& y_{1}=x_{11} / x_{10} \mapsto \lambda x_{11} /\left(\lambda^{-2} x_{10}\right)=\lambda^{3} y_{1} \\
& y_{2}=x_{12} / x_{10} \mapsto \lambda x_{12} /\left(\lambda^{-2} x_{10}\right)=\lambda^{3} y_{2} \\
& y_{3}=x_{21} / x_{20} \mapsto \lambda x_{21} /\left(\lambda^{-2} x_{20}\right)=\lambda^{3} y_{3} \\
& y_{4}=x_{22} / x_{20} \mapsto \lambda x_{22} /\left(\lambda^{-2} x_{20}\right)=\lambda^{3} y_{4} \\
& y_{5}=x_{30} / x_{31} \mapsto \lambda^{-2} x_{30} /\left(\lambda x_{31}\right)=\lambda^{-3} y_{5} \\
& y_{6}=x_{32} / x_{31} \mapsto \lambda x_{32} /\left(\lambda x_{31}\right)=y_{6} \\
& y_{7}=x_{40} / x_{42} \mapsto \lambda^{-2} x_{40} /\left(\lambda x_{42}\right)=\lambda^{-3} y_{7} \\
& y_{8}=x_{41} / x_{42} \mapsto \lambda x_{41} /\left(\lambda x_{42}\right)=y_{8} \\
& y_{9}=x_{50} / x_{51} \mapsto \lambda^{-2} x_{50} /\left(\lambda x_{51}\right)=\lambda^{-3} y_{9} \\
& y_{10}=x_{52} / x_{51} \mapsto \lambda x_{52} /\left(\lambda x_{51}\right)=y_{10} \\
& y_{11}=x_{60} / x_{61} \mapsto \lambda^{-2} x_{60} /\left(\lambda x_{61}\right)=\lambda^{-3} y_{11} \\
& y_{12}=x_{62} / x_{61} \mapsto \lambda x_{62} /\left(\lambda x_{61}\right)=y_{12}
\end{aligned}
$$

The action on the basis $\left(v_{1}, \ldots, v_{5}\right)$ of $N_{x} \cong \mathrm{C}^{5}$ is

$$
v_{1} \mapsto \lambda^{3} v_{1} ; \quad v_{2} \mapsto \lambda^{3} v_{2} ; \quad v_{3} \mapsto \lambda^{-3} v_{3} ; \quad v_{4} \mapsto \lambda^{-3} v_{4} ; \quad v_{5} \mapsto v_{5} .
$$

In this way a local model for $\left(X^{S S}(m) / / G, \xi\right)$ is $\left(\mathbb{C}^{5} / / \mathbb{C}^{*}, 0\right)$ with "weights" $(3,3,-3,-3,0)$, that is the 4 -dimensional toric variety $Y$. Using $\left(z_{1}, \ldots, z_{5}\right)$ as coordinates of $\mathrm{C}^{5}\left(\cong N_{x}\right)$ with respect to the basis $\left\{v_{1}, \ldots, v_{5}\right\}$, the ring of invariant functions is generated by

$$
T_{1}:=z_{1} z_{3}, \quad T_{2}:=z_{1} z_{4}, \quad T_{3}:=z_{2} z_{3}, \quad T_{4}:=z_{2} z_{4}, \quad T_{5}:=z_{5},
$$

and the coordinate ring of $Y$ is

$$
\mathrm{C}\left[T_{1}, \ldots, T_{5}\right] /\left(T_{1} T_{4}-T_{2} T_{3}\right) .
$$

In conclusion, the variety $\left(X^{S S}(m) / / G, \xi\right)$, where $\xi$ is a point of the curve $C_{i j} \cong \mathrm{P}^{1}(\mathrm{C})$, is locally isomorphic to the toric variety $Y$ : it is singular and there are different ways to resolve it ([2], [6]). 
$3.3-x_{i}=x_{j}, x_{h}=x_{l}, x_{k}=x_{n}$.

This study is analogous to the previous one.

Consider a polarization $m$ such that it is possible to "subdivide" it as $m_{i}+m_{j}=m_{h}+m_{l}=m_{k}+m_{n}$ (for different indexes); we are examining the configuration $x$, with $x_{i}=x_{j}, x_{h}=x_{l}, x_{k}=x_{n}$ (this configuration is a particular case of the previous one).

In the quotient $X^{S S}(m) / / G$ the image of the orbit $G x$ is a point $O_{i j, h l, k n}$ that lies on the three singular curves $C_{i j}, C_{h l}, C_{k n}$.

The orbit $G x$ is minimal, closed and strictly semi-stable: assume $x$ equal to

$$
x=\left(\begin{array}{llllll}
1 & 1 & 0 & 0 & 0 & 0 \\
0 & 0 & 1 & 1 & 0 & 0 \\
0 & 0 & 0 & 0 & 1 & 1
\end{array}\right) .
$$

Let us apply the Étale Slice Theorem as we did in the previous case: the stabilizer $G_{x}$ is isomorphic to a 2-dimensional torus $G_{x} \cong\left\{\operatorname{diag}\left(\lambda, \mu, \lambda^{-1} \mu^{-1}\right), \lambda, \mu \in \mathbb{C}^{*}\right\}$ which implies that $\operatorname{dim} G x=6$ and the space $T_{x} \mathbb{C}^{12}=\mathbb{C}^{12}$ can be decomposed $G_{x}$-invariantly as the direct sum $T_{x} G x \oplus N_{x}$, where $T_{x} G x$ and $N_{x}$ are both 6 -dimensional.

After the dehomogenization of each copy of $\mathrm{P}^{2}(\mathrm{C})$ via the unique nonzero coordinate, the configuration $x \in \mathbb{P}^{2}(\mathbb{C})^{6}$ can be considered in $\mathbb{C}^{12}$ as

$$
x=\left(\begin{array}{llllll}
0 & 0 & 0 & 0 & 0 & 0 \\
0 & 0 & 0 & 0 & 0 & 0
\end{array}\right)=(0, \ldots, 0) .
$$

Let us consider $\mathbb{C}^{12}$ with coordinates $\left(y_{1}, \ldots, y_{12}\right)$ : the equations of the 6-dimensional tangent space $T_{x} G x$ and of the 6-dimensional normal space $N_{x}$ are:

$$
\begin{aligned}
& T_{x} G x=\left\{y \in \mathbb{C}^{12} \mid \begin{array}{l}
y_{1}-y_{3}=0, \quad y_{2}-y_{4}=0, \quad y_{5}-y_{7}=0, \\
y_{6}-y_{8}=0, \quad y_{9}-y_{11}=0, \quad y_{10}-y_{12}=0
\end{array}\right\} \cong \mathbb{C}^{6}, \\
& N_{x}=\left\{y \in \mathbb{C}^{12} \mid \begin{array}{l}
y_{1}+y_{3}=0, \quad y_{2}+y_{4}=0, \quad y_{5}+y_{7}=0, \\
y_{6}+y_{8}=0, \quad y_{9}+y_{11}=0, \quad y_{10}+y_{12}=0
\end{array}\right\} \cong \mathbb{C}^{6} .
\end{aligned}
$$

Then a basis for $N_{x} \cong \mathbb{C}^{6}$ is determined by $\left\{v_{1}, \ldots, v_{6}\right\}$, where:

$$
\begin{array}{ll}
v_{1}=(1,0,-1,0,0,0,0,0,0,0,0,0), & v_{2}=(0,1,0,-1,0,0,0,0,0,0,0,0), \\
v_{3}=(0,0,0,0,1,0,-1,0,0,0,0,0), & v_{4}=(0,0,0,0,0,1,0,-1,0,0,0,0), \\
v_{5}=(0,0,0,0,0,0,0,0,1,0,-1,0), & v_{6}=(0,0,0,0,0,0,0,0,0,1,0,-1) .
\end{array}
$$

Now, by the Étale Slice Theorem, we have to study the action of the torus $\left\{\operatorname{diag}\left(\lambda, \mu, \lambda^{-1} \mu^{-1}\right), \lambda, \mu \in \mathbb{C}^{*}\right\} \cong\left(\mathbb{C}^{*}\right)^{2}$ on $N_{x}$ : this action is induced by 
the diagonal action of $S L_{3}(\mathrm{C})$ on $\mathbb{P}^{2}(\mathrm{C})^{6}(m)$. In this way on the coordinates $\left(y_{1}, \ldots, y_{12}\right)$ of $\mathrm{C}^{12}$ we have:

$\left(\lambda^{-1} \mu \cdot y_{1}, \quad \lambda^{-2} \mu^{-1} \cdot y_{2}, \quad \lambda^{-1} \mu \cdot y_{3}, \quad \lambda^{-2} \mu^{-1} \cdot y_{4}, \quad \lambda \mu^{-1} \cdot y_{5}, \quad \lambda^{-1} \mu^{-2} \cdot y_{6}\right.$, $\left.\lambda \mu^{-1} \cdot y_{7}, \quad \lambda^{-1} \mu^{-2} \cdot y_{8}, \quad \lambda^{2} \mu \cdot y_{9}, \quad \lambda \mu^{2} y_{10}, \quad \lambda^{2} \mu \cdot y_{11}, \quad \lambda \mu^{2} \cdot y_{12}\right)$.

The action on the basis $\left(v_{1}, \ldots, v_{6}\right)$ of $N_{x} \cong \mathrm{C}^{6}$ is

$$
\begin{array}{lll}
v_{1} \mapsto \lambda^{-1} \mu \cdot v_{1} ; & v_{2} \mapsto \lambda^{-2} \mu^{-1} \cdot v_{2} ; & v_{3} \mapsto \lambda \mu^{-1} \cdot v_{3} ; \\
v_{4} \mapsto \lambda^{-1} \mu^{-2} \cdot v_{4} ; & v_{5} \mapsto \lambda^{2} \mu \cdot v_{5} ; & v_{6} \mapsto \lambda \mu^{2} \cdot v_{6} .
\end{array}
$$

It follows that a local model for $\left(X^{S S}(m) / / G, O_{i j, h l, k n}\right)$ is given by $Y:=\left(\mathbb{C}^{6} / /\left(\mathbb{C}^{*}\right)^{2}, 0\right)$, where the action of $\left(\mathbb{C}^{*}\right)^{2}$ can be written (in the coordinates $\left(z_{1}, \ldots, z_{6}\right)$ of $\left.N_{x} \cong \mathbb{C}^{6}\right)$ as

$$
(\lambda, \mu)\left(z_{1}, \ldots, z_{6}\right) \rightarrow\left(\lambda^{-1} \mu z_{1}, \lambda^{-2} \mu^{-1} z_{2}, \lambda \mu^{-1} z_{3}, \lambda^{-1} \mu^{-2} z_{4}, \lambda^{2} \mu z_{5}, \lambda \mu^{2} z_{6}\right) .
$$

Thus we obtain a 4-dimensional toric variety $Y$ : the ring of invariant functions is generated by

$$
T_{1}:=z_{1} z_{3}, \quad T_{2}:=z_{2} z_{5}, \quad T_{3}:=z_{4} z_{6}, \quad T_{4}:=z_{1} z_{4} z_{5}, \quad T_{5}:=z_{2} z_{3} z_{6},
$$

and the coordinate ring of $Y$ is:

$$
\text { C }\left[T_{1}, \ldots, T_{5}\right] /\left(T_{1} T_{2} T_{3}-T_{4} T_{5}\right) .
$$

Its singular locus is given by three lines $s_{1}=\{(t, 0,0,0,0), t \in \mathbb{C}\}$, $s_{2}=\{(0, t, 0,0,0), t \in \mathbb{C}\}$ and $s_{3}=\{(0,0, t, 0,0), t \in \mathbb{C}\}$ that have a common point, the origin. These lines correspond to the curves $C_{i j}, C_{h l}, C_{k n}$.

A toric representation of $Y$ is determined by a rational, polyhedral cone $\sigma \subset \mathbb{R}^{4}$, such that $\operatorname{Spec}\left(\sigma^{\vee} \cap Z^{4}\right) \cong Y$. The generators of the semi-group $\sigma^{\vee} \cap Z^{4}$ are $w_{1}, \ldots, w_{5} \in Z^{4}$ and satisfy $w_{1}+w_{2}+w_{3}=w_{4}+w_{5}$. Assume

$$
\begin{gathered}
w_{1}=(1,0,0,0), \quad w_{2}=(0,1,0,0), \quad w_{3}=(0,0,1,0), \\
w_{4}=(0,0,0,1), \quad w_{5}=(1,1,1,-1) .
\end{gathered}
$$

The primitive elements of $\sigma$ are:

$$
\begin{array}{lll}
\boldsymbol{n}_{1}=(0,0,1,1), & \boldsymbol{n}_{2}=(1,0,0,0), & \boldsymbol{n}_{3}=(0,0,1,0), \\
\boldsymbol{n}_{4}=(0,1,0,1), & \boldsymbol{n}_{5}=(1,0,0,1), & \boldsymbol{n}_{6}=(0,1,0,0) .
\end{array}
$$

It is clear that the cone $\sigma$ is singular.

Let us intesect $\sigma$ with a transversal hyperplane $\pi$ of $\mathbb{R}^{4}$ and then consider the projection on $\pi$. With $\pi: y_{1}+y_{2}+y_{3}+y_{4}=2$ we get the poly- 
tope $\Pi$ of $\mathbb{R}^{3}$, with vertices

$$
\begin{array}{lll}
u_{1}=(0,0,1), & u_{2}=(2,0,0), & u_{3}=(0,0,2), \\
u_{4}=(0,1,0), & u_{5}=(1,0,0), & u_{6}=(0,2,0) .
\end{array}
$$

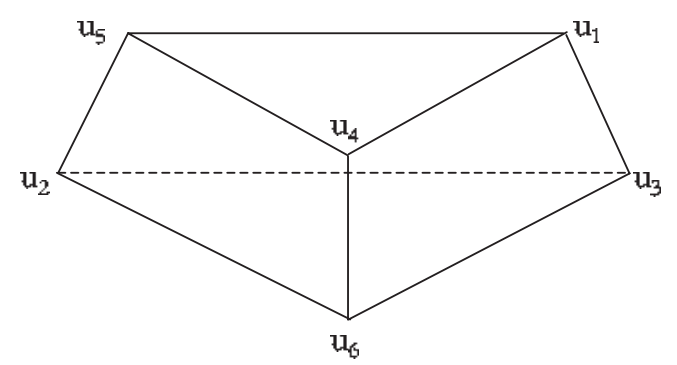

Fig. Polytope $\Pi$.

In conclusion the pointed variety $\left(X^{S S}(m) / / G, O_{i j, h l, k n}\right)$ is isomorphic to the toric variety $\mathbb{C}\left[T_{1}, \ldots, T_{5}\right] /\left(T_{1} T_{2} T_{3}-T_{4} T_{5}\right)$, where the action has weights

$$
\left(\begin{array}{cccccc}
-1 & -2 & 1 & -1 & 2 & 1 \\
1 & -1 & -1 & -2 & 1 & 2
\end{array}\right)
$$

$3.4-x_{h}=x_{i}=x_{j}$ and $x_{k}, x_{l}, x_{n}$ collinear.

Consider a polarization $m$ such that $m_{h}+m_{i}+m_{j}=|m| / 3$ and $m_{k}+m_{l}+m_{n}=2|m| / 3$ (for different indexes); then let us study the configuration $x$ where: $x_{h}=x_{i}=x_{j}$ and $x_{k}, x_{l}, x_{n}$ collinear.

The orbit $G x$ is minimal, closed, strictly semi-stable and its image in $X^{S S}(m) / / G$ is a point $O_{h i j}$. In particular $x_{k}, x_{l}, x_{n}$ have to be all distinct.

As in the previous cases, by the Étale Slice Theorem, we obtain a local model for $\left(X^{S S}(m) / / G, O_{h i j}\right)$ : this is determined by $Y:=\left(\mathbb{C}^{5} / / \mathbb{C}^{*}, 0\right)$, where the action of $\mathbb{C}^{*}$ over $\mathbb{C}^{5}$ with coordinate $\left(z_{1}, \ldots, z_{5}\right)$ has weights $(3,3,3,3,-3) . Y$ is a 4 -dimensional toric variety that corresponds to the smooth affine variety with coordinate ring

$$
\mathbb{C}\left[T_{1}, \ldots, T_{4}\right] \cong \mathbb{C}^{4}
$$

In conclusion the corresponding point $O_{h i j}$ in $X^{S S}(m) / / G$ is nonsingular.

We have classified the different singularities of $X^{S S}(m) / / G$ : 
TheOREM 3.9. Let $X=\mathbb{P}^{2}(\mathbb{C})^{6}$ and $m \in Z_{>0}^{6}$ a polarization:

1. $m$ s.t.

$-3 \nmid|m|$,

- $m_{i}<|m| / 3 \forall i$

then the quotient $X^{S S}(m) / / G=X^{S}(m) / G$ is geometric and nonsingular;

2. $m$ s.t.

-3||$m \mid$,

- $m_{i}<|m| / 3 \forall i$,

- for all couples and triples of indexes we have $m_{i}+m_{j} \neq|m| / 3$ or $m_{h}+m_{i}+m_{j} \neq|m| / 3$,

then the quotient $X^{S S}(m) / / G=X^{S}(m) / G$ is geometric and nonsingular;

3. $m$ s.t.

-3||$m \mid$,

- there exists an index $i$ s.t. $m_{i}=|m| / 3$, while for the other indexes $j \neq i, m_{j}<|m| / 3$,

then the quotient is $\left(\mathbb{P}^{1}(\mathrm{C})\right)^{5}\left(m^{\prime}\right) / / S L_{2}(\mathrm{C})$; its dimension is equal to two, and the polarization $m^{\prime} \in Z_{>0}^{5}$ is obtained from $m$ by eliminating $m_{i}$;

4. $m$ s.t.

-3||$m \mid$,

- there exist two different indexes $i, j$ s.t. $m_{i}=m_{j}=|m| / 3$, while for the others $h \neq i, j, m_{h}<|m| / 3$,

then the quotient is $\left(\mathbb{P}^{1}(\mathbb{C})\right)^{4}\left(m^{\prime \prime}\right) / / S L_{2}(\mathbb{C}) \cong \mathbb{P}^{1}(\mathbb{C})$; the polarization $m^{\prime \prime} \in Z^{4}$ is obtained from $m$ by eliminating $m_{i}$ and $m_{j}$;

5. $m$ s.t.

-3||$m \mid$

- $m_{i}<|m| / 3 \forall i$,

- there are two different indexes $i, j$ s.t. $m_{i}+m_{j}=|m| / 3$,

then the categorical quotient $X^{S S}(m) / / G$ includes a curve $C_{i j} \cong \mathbb{P}^{1}(\mathbb{C})$, that corresponds to strictly semi-stable orbits s.t. $x_{i}=x_{j}$ or $x_{h}, x_{k}, x_{l}, x_{n}$ collinear. In particular points $\xi$ of $C_{i j}$ are singular: 
locally, the variety $\left(X^{S S}(m) / / G, \xi\right)$ is isomorphic to the toric variety

$$
\text { C }\left[T_{1}, T_{2}, T_{3}, T_{4}, T_{5}\right] /\left(T_{1} T_{4}-T_{2} T_{3}\right) .
$$

6. $m$ s.t.

-3||$m \mid$

- $m_{i}<|m| / 3 \forall i$,

- there is a "partition" of $m$ such that $m_{i}+m_{j}=m_{h}+m_{l}=$ $=m_{k}+m_{n}$,

then the categorical quotient $X^{S S}(m) / / G$ includes three curves $C_{i j}, C_{h l}$, $C_{k n} \cong \mathrm{P}^{1}(\mathrm{C})$, that have a common point $O_{i j, h l, k n}$.

In particular $O_{i j, h l, k n}$ is singular: locally the variety $\left(X^{S S}(m) / / G, O_{i j, h l, k n}\right)$ is isomorphic to the toric variety

$$
\text { C }\left[T_{1}, T_{2}, T_{3}, T_{4}, T_{5}\right] /\left(T_{1} T_{2} T_{3}-T_{4} T_{5}\right) .
$$

7. $m$ s.t.

-3||$m \mid$

- $m_{i}<|m| / 3 \forall i$,

- there are three indexes $h, i, j$ s.t. $m_{h}+m_{i}+m_{j}=|m| / 3$,

then the categorical quotient $X^{S S}(m) / / G$ includes a point $O_{\text {hij }}$ that corresponds to the minimal, closed, strictly semi-stable orbit Gx such that $x_{h}=x_{i}=x_{j}$ and $x_{k}, x_{l}, x_{n}$ are collinear. The point $O_{\text {hij }}$ is non singular.

\section{$3.5-$ Examples.}

Now we provide two examples that illustrate how to get explicitly a quotient, via its coordinate ring, or via an elementary transformation.

$3.6-\mathbb{P}^{2}(\mathbb{C})^{6}(222111)$

$|m|=9$. Since $m_{i}<|m| / 3$ for all $i$ and, for example, $m_{1}+m_{4}=|m| / 3$, we have $X^{S}(m) \neq \emptyset$ and $X^{S}(m) \subsetneq X^{S S}(m)$.

Moreover it is easy to verify that there are nine $C_{i j}$ curves, six $O_{i j, h l, k n}$ points and one $O_{h i j}$ point $\left(O_{456}\right)$.

Let us study the graded algebra of $G$-invariant functions $R_{2}^{6}(m)^{G}$. A standard tableau $\tau$ of degree $k$ associated to the polarization $m$ looks like 


$$
\left.\tau=\left[\begin{array}{lll}
a_{1}^{1} & a_{2}^{2} & a_{3}^{3} \\
a_{2}^{1} & a_{3}^{2} & a_{4}^{3} \\
a_{3}^{1} & a_{4}^{2} & a_{5}^{3} \\
a_{4}^{1} & a_{5}^{2} & a_{6}^{3}
\end{array}\right]\right\} 3 k
$$

where $a_{j}^{i}$ denotes a column vector in the $i$-th column of $\tau$ with all coordinates equal to $j$. If $\left|a_{j}^{i}\right|$ is the length of $a_{j}^{i}$, we have

$$
\begin{array}{lll}
\left|a_{1}^{1}\right|=2 k, & \left|a_{6}^{3}\right|=k, & \left|a_{2}^{1}\right|+\left|a_{2}^{2}\right|=2 k, \\
\left|a_{3}^{1}\right|+\left|a_{3}^{2}\right|+\left|a_{3}^{3}\right|=2 k, & \left|a_{4}^{1}\right|+\left|a_{4}^{2}\right|+\left|a_{4}^{3}\right|=k, & \left|a_{5}^{2}\right|+\left|a_{5}^{3}\right|=k, \\
\sum_{i=2}^{4}\left|a_{i}^{1}\right|=k, & \sum_{i=2}^{5}\left|a_{i}^{2}\right|=3 k, & \sum_{i=3}^{5}\left|a_{i}^{3}\right|=2 k .
\end{array}
$$

Let $\alpha_{3}:=\left|a_{3}^{1}\right|, \alpha_{4}:=\left|a_{4}^{1}\right|, \beta_{3}:=\left|a_{3}^{3}\right|, \beta_{4}:=\left|a_{4}^{3}\right|$. Then it follows that:

$$
\begin{array}{lll}
\left|a_{1}^{1}\right|=2 k, & \left|a_{2}^{2}\right|=k+\alpha_{3}+\alpha_{4}, & \left|a_{3}^{3}\right|=\beta_{3}, \\
\left|a_{2}^{1}\right|=k-\left(\alpha_{3}+\alpha_{4}\right), & \left|a_{3}^{2}\right|=2 k-\left(\alpha_{3}+\beta_{3}\right), & \left|a_{4}^{3}\right|=\beta_{4}, \\
\left|a_{3}^{1}\right|=\alpha_{3}, & \left|a_{4}^{2}\right|=k-\left(\alpha_{4}+\beta_{4}\right), & \left|a_{5}^{3}\right|=2 k-\left(\beta_{3}+\beta_{4}\right), \\
\left|a_{4}^{1}\right|=\alpha_{4}, & \left|a_{5}^{2}\right|=\beta_{3}+\beta_{4}-k, & \left|a_{6}^{3}\right|=k .
\end{array}
$$

Moreover $\alpha_{3}, \alpha_{4}, \beta_{3}, \beta_{4}$ must satisfy the inequalities:

$$
\begin{array}{lll}
0 \leq \alpha_{3}, \alpha_{4}, \beta_{3}, \beta_{4} \leq 2 k, & \alpha_{3}+2 \alpha_{4} \leq \beta_{3}, & \alpha_{3}+\alpha_{4} \leq k, \\
k+\alpha_{4} \leq \beta_{3}+\beta_{4} \leq 2 k, & \beta_{3} \leq k+\alpha_{3}+\alpha_{4}, & 2 \beta_{3}+\beta_{4} \leq 3 k+\alpha_{4} .
\end{array}
$$

Assume

$$
x:=\alpha_{4}, \quad y:=\alpha_{3}+\alpha_{4}, \quad z:=\beta_{3}, \quad w:=\beta_{3}+\beta_{4} ;
$$

the standard tableau $\tau$ (15) is completely determined by the vector $(x, y, z, w)$ that satisfies:

$$
\begin{array}{ccl}
0 \leq x \leq y \leq k, \quad 0 \leq z \leq w \leq 2 k, & 0 \leq y+z-x \leq 2 k, \\
x+y \leq z \leq y+k, \quad z \leq w \leq k+z, & 0 \leq w+x-z \leq k, \quad w \geq x+k .
\end{array}
$$

After a few calculations we find out that for any $k$, there are

$$
\frac{1}{8}\left(k^{4}+6 k^{3}+15 k^{2}+18 k\right)+1\left(=\operatorname{dim}\left(R_{2}^{6}(m)_{k}^{G}\right)\right)
$$

standard tableaux. Thus the Hilbert function of the graded $\operatorname{ring} R_{2}^{6}(m)^{G}$ is 
equal to

$$
\sum_{k=0}^{\infty}\left(\frac{1}{8}\left(k^{4}+6 k^{3}+15 k^{2}+18 k\right)+1\right) t^{k}=\frac{1-t^{3}}{(1-t)^{6}}
$$

This suggests that the quotient $X^{S S}(m) / / G$ is isomorphic to a cubic hypersurface in $\mathbb{P}^{5}(\mathbb{C})$.

By the First Fundamental Theorem of Invariant Theory we know that the algebra of invariants $R_{2}^{6}(m)^{G}$ is generated by bracket functions: to each tableau we can associate a tableau function that is a product of bracket functions. Let us see in details. A configuration $x \in X$ can be written has a matrix $3 \times 6$

$$
x=\left(\begin{array}{llll}
x_{01} & x_{02} & \ldots & x_{06} \\
x_{11} & x_{12} & \ldots & x_{16} \\
x_{21} & x_{22} & \ldots & x_{26}
\end{array}\right)
$$

where the $i$-th column contains the coordinates of the $i$-th point. The bracket function $\operatorname{det}_{j_{1} j_{2} j_{3}}$ on the space of $3 \times 6$ matrices is equal to the maximal minor of $x$ formed by the columns $j_{1}, j_{2}$ and $j_{3}: \operatorname{det}_{j_{1} j_{2} j_{3}}(x)=$ $=\left[j_{1} j_{2} j_{3}\right]$.

Now for each tableau $\tau=\left[\begin{array}{ccc}\tau_{11} & \tau_{12} & \tau_{13} \\ \ldots & \ldots & \ldots \\ \tau_{r 1} & \tau_{r 2} & \tau_{r 3}\end{array}\right]$ with $r$ rows and 3 columns, we
fine the tableau function

$$
t=\prod_{i=1}^{r}\left[\begin{array}{lll}
\tau_{i 1} & \tau_{i 2} & \tau_{i 3}
\end{array}\right]
$$

In particular, each such function is an invariant for the group $S L_{3}(\mathrm{C})$.

Now for $m=(222111)$ we have six standard tableaux $\tau_{i}$ of degree $k=1$ :

$$
\begin{aligned}
& \tau_{0}=\left[\begin{array}{l}
124 \\
135 \\
236
\end{array}\right], \quad \tau_{1}=\left[\begin{array}{l}
123 \\
135 \\
246
\end{array}\right], \quad \tau_{2}=\left[\begin{array}{l}
123 \\
134 \\
256
\end{array}\right], \\
& \tau_{3}=\left[\begin{array}{l}
123 \\
125 \\
346
\end{array}\right], \quad \tau_{4}=\left[\begin{array}{l}
123 \\
124 \\
356
\end{array}\right], \quad \tau_{5}=\left[\begin{array}{l}
123 \\
123 \\
456
\end{array}\right] .
\end{aligned}
$$

Then we get the following invariants of $R_{2}^{6}(m)^{G}$ :

$$
\begin{aligned}
t_{0} & =[124][135][236], & t_{1}=[123][135][246], & t_{2}=[123][134][256], \\
t_{3} & =[123][125][346], & t_{4}=[123][124][356], & t_{5}=[123][123][456] .
\end{aligned}
$$


For every $(i, j) \neq(2,3),(3,2)$, the product $t_{i} t_{j}$ is a standard tableau function from $R_{2}^{6}(m)_{2}^{G}$. Applying the straightening algorithm (that allows one to write any tableau function as a linear combination of standard tableau functions), we obtain:

$$
t_{2} t_{3}=t_{1} t_{4}-u+t_{5}\left(-t_{0}+t_{1}-t_{2}-t_{3}+t_{4}-t_{5}\right) .
$$

So the standard monomial $u=[123][123][123][145][246][356]$ can be expressed as a polynomial of degree two in the $t_{i}$.

If we take a tableau function $t_{(x, y, z, w, k)}$ corresponding to a standard tableau $\tau_{(x, y, z, w, k)}(15)$, we can write it as

$$
t_{(x, y, z, w, k)}= \begin{cases}t_{0}^{k+x-z} t_{1}^{k+z-x-w} t_{2}^{w-y-k} t_{4}^{y-x} t_{5}^{x}, & z \leq x+k, w \leq k+z-x ; \\ t_{0}^{k+x-z} t_{1}^{z-x-y} t_{3}^{k+y-w} t_{4}^{w-x-k} t_{5}^{x}, & z \leq x+k, y \leq z-x ; \\ t_{1}^{3 k+x-w-z} t_{2}^{w-y-k} t_{4}^{k+y-z} t_{5}^{x} u^{z-x-k}, & z \geq x+k, w \leq 3 k+x-z ; \\ t_{1}^{2 k+x-y-z} t_{3}^{k+y-w} t_{4}^{w-z} t_{5}^{x} u^{z-x-k}, & z \geq x+k, y \leq 2 k+x-z .\end{cases}
$$

In other words $t_{0}, \ldots, t_{5}$ are the generators of $R_{2}^{6}(m)^{G}$.

Applying the straightening algorithm to the non-standard product $t_{0} u$, we have:

$$
t_{0} u=t_{1} t_{4}\left(t_{1}-t_{2}-t_{3}+t_{4}-t_{5}\right) .
$$

Then by relation (16), it follows that

$$
\begin{gathered}
t_{0}\left(t_{1} t_{4}-t_{2} t_{3}+t_{5}\left(-t_{0}+t_{1}-t_{2}-t_{3}+t_{4}-t_{5}\right)\right)=t_{1} t_{4}\left(t_{1}-t_{2}-t_{3}+t_{4}-t_{5}\right) \Rightarrow \\
t_{0}\left(-t_{2} t_{3}+t_{5}\left(-t_{0}+t_{1}-t_{2}-t_{3}+t_{4}-t_{5}\right)\right)=t_{1} t_{4}\left(-t_{0}+t_{1}-t_{2}-t_{3}+t_{4}-t_{5}\right) \Rightarrow \\
\left(-t_{0}+t_{1}-t_{2}-t_{3}+t_{4}-t_{5}\right)\left(t_{0} t_{5}-t_{1} t_{4}\right)-t_{0} t_{2} t_{3}=0
\end{gathered}
$$

Let

$$
F_{3}=\left(-T_{0}+T_{1}-T_{2}-T_{3}+T_{4}-T_{5}\right)\left(T_{0} T_{5}-T_{1} T_{4}\right)-T_{0} T_{2} T_{3},
$$

there is a surjective homomorphism of the graded algebras

$$
\mathrm{C}\left[T_{0}, T_{1}, T_{2}, T_{3}, T_{4}, T_{5}\right] /\left(F_{3}\left(T_{0}, T_{1}, T_{2}, T_{3}, T_{4}, T_{5}\right)\right) \longrightarrow R_{2}^{6}(m)^{G} .
$$

Thus the quotient $X^{S S}(m) / / G$ is isomorphic to the cubic hypersurface $F_{3}\left(T_{0}, T_{1}, T_{2}, T_{3}, T_{4}, T_{5}\right)=0$.

We want to verify that its singular locus is given by nine $C_{i j}$ curves with six $O_{i j, h l, k n}$ points; moreover we want to identify the non-singular $O_{h i j}$ point.

Using the software Reduce, we find that the singular locus of $X^{S S}(m) / / G$ corresponds to nine projective lines $r_{i}$ : these lines meet three 
by three in six "special" points $A_{k}$ : each line contains two "special" points.

$$
\begin{aligned}
& r_{1}:\left\{T_{0}-T_{4}=0, T_{1}-T_{5}=0, T_{2}=T_{3}=0\right\} ; \\
& r_{2}:\left\{T_{0}-T_{1}=0, T_{0}-T_{4}=0, T_{2}-T_{3}=0, T_{2}-T_{4}+T_{5}=0\right\} ; \\
& r_{3}:\left\{T_{0}=T_{1}=T_{3}=0, T_{2}-T_{4}+T_{5}=0\right\} ; \\
& r_{4}:\left\{T_{0}=T_{1}=T_{2}=0, T_{3}-T_{4}+T_{5}=0\right\} ; \\
& r_{5}:\left\{T_{0}=T_{1}=T_{4}=0, T_{2}+T_{5}=0\right\} ; \\
& r_{6}:\left\{T_{0}=T_{2}=T_{4}=0, T_{1}-T_{3}-T_{5}=0\right\} \\
& r_{7}:\left\{T_{0}=T_{1}=T_{4}=0, T_{3}+T_{5}=0\right\} \\
& r_{8}:\left\{T_{0}=T_{3}=T_{4}=0, T_{1}-T_{2}-T_{5}=0\right\} \\
& r_{9}:\left\{T_{0}-T_{1}=0, T_{2}=T_{3}=0, T_{4}-T_{5}=0\right\} .
\end{aligned}
$$

"Special” points:

$$
\begin{array}{ll}
A_{1}=[1: 1: 0: 0: 1: 1] ; & A_{1} \in r_{1}, r_{2}, r_{9} ; \\
A_{2}=[0: 1: 0: 0: 0: 1] ; & A_{2} \in r_{1}, r_{6}, r_{8} \\
A_{3}=[0: 0: 1: 1: 0:-1] ; & A_{3} \in r_{2}, r_{5}, r_{7} \\
A_{4}=[0: 0: 1: 0: 0:-1] ; & A_{4} \in r_{3}, r_{5}, r_{8} \\
A_{5}=[0: 0: 0: 0: 1: 1] ; & A_{5} \in r_{3}, r_{4}, r_{9} \\
A_{6}=[0: 0: 0: 1: 0:-1] ; & A_{6} \in r_{4}, r_{6}, r_{7} .
\end{array}
$$

The lines $r_{1}, \ldots, r_{9}$ lie in $\left(X^{S S}(m) / / G\right) \backslash\left(X^{S}(m) / G\right)$; they correspond to the nine curves $C_{i j} \cong \mathbb{P}^{1}(\mathbb{C})$ : for example, $r_{1} \equiv C_{25}$, because

$$
\begin{gathered}
{[124][135][236]-[123][124][356]=0,[123][135][246]-[123][123][456]=0,} \\
{[124][134][256]=[123][125][346]=0 .}
\end{gathered}
$$

In the same way

$$
\begin{array}{llll}
r_{2} \equiv C_{16}, & r_{3} \equiv C_{15}, & r_{4} \equiv C_{26}, & r_{5} \equiv C_{24} \\
r_{6} \equiv C_{14}, & r_{7} \equiv C_{35}, & r_{8} \equiv C_{36}, & r_{9} \equiv C_{34}
\end{array}
$$

The points $A_{1}, \ldots, A_{6}$ correspond to the points $O_{i j, h l, k n}$ with $i, h, k \in$ $\in\{1,2,3\}$ and $j, l, n \in\{4,5,6\}$ :

$$
\begin{array}{lll}
A_{1}=O_{16,25,34}, & A_{2}=O_{14,25,36}, & A_{3}=O_{16,24,35}, \\
A_{4}=O_{15,24,36}, & A_{5}=O_{15,26,34}, & A_{6}=O_{14,26,35} .
\end{array}
$$

Finally the point $O_{456} \in X^{S S}(m) / / G$ corresponds to $[1: 0: 0: 0: 0: 0]$ which is non-singular.

\section{$3.7-\mathbb{P}^{2}(\mathbb{C})^{6}(221111)$.}

$|\widehat{m}|=8$; since $|\widehat{m}|$ is not divisible by 3 , we have $X^{S}(\widehat{m})=X^{S S}(\widehat{m})$. 
In order to determine this geometric quotient, we have to introduce the elementary transformation $\widehat{m}=(221111) \stackrel{+1_{3}}{\longrightarrow}(222111)=m$, and consequently

$$
\widehat{\theta}: X^{S}(\widehat{m}) / G \longrightarrow X^{S S}(m) / / G .
$$

First of all let us study $\widehat{\theta}^{-1}\left(O_{456}\right)$ : by relation (6) its dimension is equal to $d=3$; the semi-stable orbits of $X^{S S}(m)$ that determine $O_{456}$ in the quotient $X^{S S}(m) / / G$ and are included in $X^{S}(\widehat{m})$, are characterized by $x_{1}, x_{2}, x_{3}$ collinear. Each orbit of this type contains a point of the form

$$
\left(\begin{array}{cccccc}
1 & 0 & 1 & 0 & a_{1} & a_{2} \\
0 & 1 & 1 & 0 & b_{1} & b_{2} \\
0 & 0 & 0 & 1 & 1 & 1
\end{array}\right)
$$

where $\left(a_{1}, b_{1}, a_{2}, b_{2}\right) \in \mathbb{C}^{4} \backslash\{0\}$. Two such points belong to the same orbit if and only if they differ by the action of the group $\left\{\left(\lambda, \lambda, \lambda^{-2}\right)\right\}$, i.e. the point above is in the same orbit as

$$
\left(\begin{array}{cccccc}
1 & 0 & 1 & 0 & \lambda^{3} a_{1} & \lambda^{3} a_{2} \\
0 & 1 & 1 & 0 & \lambda^{3} b_{1} & \lambda^{3} b_{2} \\
0 & 0 & 0 & 1 & 1 & 1
\end{array}\right) .
$$

It follows that $\widehat{\theta}^{-1}\left(O_{456}\right) \cong \mathbb{P}^{3}(\mathrm{C})$.

Then $\widehat{\theta}^{-1}(\xi), \xi \in C_{i j}$; studying how semi-stable orbits change going from $X^{S S}(m)$ to $X^{S}(\widehat{m})$, there can be two different cases: coincidence or collinearity.

1. Consider the curve $C_{14}$ : by the numerical criterion for $X^{S}(\widehat{m})$, orbits which have $x_{2}, x_{3}, x_{5}, x_{6}$ collinear are stable. In particular by relation (6), the dimension of $\widehat{\theta}^{-1}\left(\xi_{1}\right), \xi_{1} \in C_{14}$ is equal to $d=1$ : in fact

$$
\widehat{\theta}^{-1}\left(\xi_{1}\right) \cong \mathbb{P}^{1}(\mathbb{C}) \text {. }
$$

2. Consider the curve $C_{36}$ : by the numerical criterion for $X^{S}(\widehat{m})$ orbits which have $x_{3}=x_{6}$ are stable. In particular by relation (5), the dimension of $\widehat{\theta}^{-1}\left(\xi_{2}\right), \xi_{2} \in C_{36}$ is equal to $d=1$; in fact

$$
\widehat{\theta}^{-1}\left(\xi_{2}\right) \cong \mathbb{P}^{1}(\mathbb{C}) \text {. }
$$

Let us study $\widehat{\theta}^{-1}\left(O_{i j, h l, k n}\right)$; consider $O_{14,25,36}$. Strictly semi-stable orbits that contain the orbit $G x\left(x_{1}=x_{4}, x_{2}=x_{5}, x_{3}=x_{6}\right)$ in their closure, are characterized by one of the following properties: 
1. $x_{1}=x_{4}$ and $x_{1}, x_{2}, x_{5}$ collinear; $\quad 2 . x_{1}=x_{4}$ and $x_{1}, x_{3}, x_{6}$ collinear;

3. $x_{2}=x_{5}$ and $x_{1}, x_{2}, x_{4}$ collinear; $\quad 4 . x_{2}=x_{5}$ and $x_{2}, x_{3}, x_{6}$ collinear;

5. $x_{3}=x_{6}$ and $x_{1}, x_{3}, x_{4}$ collinear; $\quad 6 . x_{3}=x_{6}$ and $x_{2}, x_{3}, x_{5}$ collinear.

In particular configurations $1,2,3,4$ are unstable for the polarization $\widehat{m}$, while 5 and 6 are included in $X^{S}(\widehat{m})$; moreover these sets have a common configuration: ( $x_{3}=x_{6}, x_{1}, x_{3}, x_{4}$ collinear, $x_{2}, x_{3}, x_{5}$ collinear):

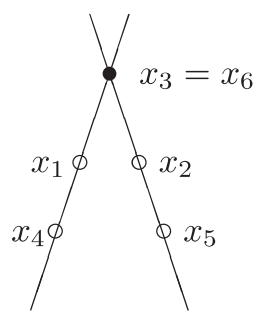

Each one of these two sets of stable configurations determines a copy of $\mathbb{P}^{1}(\mathrm{C})$ in the quotient $X^{S}(\widehat{m}) / G$ : thus these two copies of $\mathbb{P}^{1}(\mathrm{C})$ have a common point.

$$
\widehat{\theta}^{-1}\left(O_{i j, h l, k n}\right) \cong \mathbb{P}^{1}(\mathrm{C}) \cup \mathbb{P}^{1}(\mathrm{C}) \text { with a common point. }
$$

We can get this result in a different way, by constructing a subdivision of the polytope $\Pi$ (figure 2 ).

Since $X^{U S}(m) \subset X^{U S}(\widehat{m})$ and $\left(X^{U S}(\widehat{m}) \backslash X^{U S}(m)\right) \subset X^{S S S}(m)$, we determine (locally in $N_{x}$ ), which strictly semi-stable orbits for the polarization $m$ are unstable for $\widehat{m}$. By the machinery of the theory of homogeneous coordinates for a toric variety ([1],[2], [3]), the local resolution of $\left(X^{S S}(m) / / G, O_{14.25 .36}\right) \cong\left(\mathbb{C}^{6} /\left(\mathbb{C}^{*}\right)^{2}, 0\right)$ in the quotient $X^{S}(\widehat{m}) / G$ is determined by $\left(\mathbb{C}^{6} \backslash Z\right) / / H$, where $\mathbb{C}^{6} \backslash Z=\mathbb{C}^{6} \backslash\left\{z \in \mathbb{C}^{6} \mid z_{1} z_{4}=0\right.$, $\left.z_{2} z_{3}=0, z_{2} z_{4}=0\right\}$, and $H$ is the 2-dimensional torus $H=\left\{\left(\lambda_{1}, \lambda_{2}, \lambda_{1}^{-1}\right.\right.$, $\left.\left.\lambda_{1}^{-1} \lambda_{2}, \lambda_{2}^{-1}, \lambda_{1} \lambda_{2}^{-1}\right), \lambda_{1}, \lambda_{2} \in \mathbb{C}^{*}\right\}$.

The set $\mathbb{C}^{6} \backslash Z$ describes a particular resolution of $\Pi$

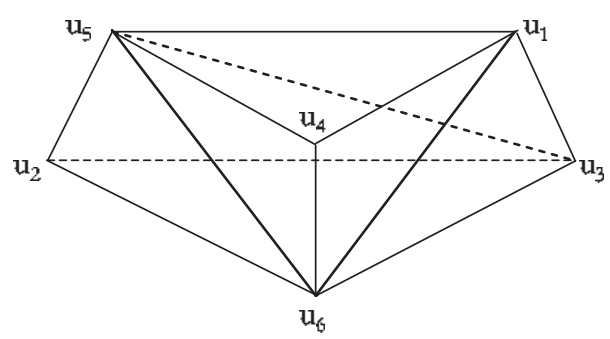

Fig. 3. - Subdivision of type (221111) of $\Pi$. 
We can find three simplicial polytopes: figure 4.

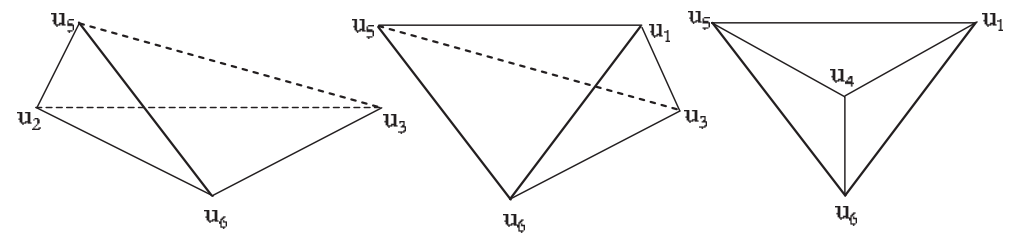

Fig. 4. - The three polytopes of the subdivision (221111) of $\Pi$.

The toric representation of $Y$, described by the polytope $\Pi$, is determined by the cone $\sigma$ : to solve its singularities let us construct a fan $\Sigma$, refinement of $\sigma$. By the theory of toric varieties, there exists a proper, birational morphism $\varphi$

$$
X_{\Sigma} \cong\left(\mathbb{C}^{6} \backslash Z\right) / / H \cong\left(\mathbb{C}^{6} \backslash Z\right) / /\left(\mathbb{C}^{*}\right)^{2} \stackrel{\varphi}{\longrightarrow}\left(\mathbb{C}^{6} / /\left(\mathbb{C}^{*}\right)^{2}\right) \cong\left(N_{x} / / G_{x}\right) \cong X_{\sigma},
$$

induced by the identity over the lattice $\mathbb{R}^{4}$ : this application allows us to specify the map $\widehat{\theta}$ :

$$
\widehat{\theta}: X^{S}(\widehat{m}) / G \longrightarrow X^{S S}(m) / / G .
$$

First of all let us take a cover of $\left(\mathbb{C}^{6} \backslash Z\right)$ : for example the three open sets $U_{1}, U_{2}, U_{3}$ :

$$
\begin{gathered}
U_{1}=\mathbb{C}^{6} \backslash\left\{z \in \mathbb{C}^{6} \mid z_{1} z_{4}=0\right\} ; \quad U_{2}=\mathbb{C}^{6} \backslash\left\{z \in \mathbb{C}^{6} \mid z_{2} z_{3}=0\right\} ; \\
U_{3}=\mathbb{C}^{6} \backslash\left\{z \in \mathbb{C}^{6} \mid z_{2} z_{4}=0\right\} .
\end{gathered}
$$

Now let us consider the action of $H \cong\left(\mathbb{C}^{*}\right)^{2}$ on these three open sets and construct the three quotients: in the first case, the quotient $\widetilde{U_{1}}=U_{1} / / H$ is the smooth variety $\mathrm{C}\left[X_{1}, X_{2}, X_{3}, X_{4}, X_{6}\right] /\left(X_{2}-X_{4} X_{6}\right)$.

In the same way $\widetilde{U}_{2}=U_{2} / / H=\mathrm{C}\left[Y_{1}, Y_{2}, Y_{3}, Y_{5}, Y_{7}\right] /\left(Y_{3}-Y_{5} Y_{7}\right)$ and $\widetilde{U}_{3}=U_{3} / / H=\mathbb{C}\left[Z_{1}, Z_{2}, Z_{3}, Z_{8}, Z_{9}\right] /\left(Z_{1}-Z_{8} Z_{9}\right)$.

How do these quotients $\widetilde{U}_{i}(i=1,2,3)$ fit together? We have the following "gluing"

$$
\begin{array}{lll}
X_{1}=Y_{1}=Z_{8} Z_{9} & Y_{1}=X_{1}=Z_{8} Z_{9} & Z_{2}=X_{4} X_{6}=Y_{2} \\
X_{3}=Y_{5} Y_{7}=Z_{3} & Y_{2}=X_{4} X_{6}=Z_{2} & Z_{3}=X_{3}=Y_{5} Y_{7} \\
X_{4}=Y_{1} Y_{2} Y_{7}=Z_{2} Z_{8} & Y_{5}=X_{1} X_{3} X_{6}=Z_{3} Z_{8} & Z_{8}=X_{6}^{-1}=Y_{1} Y_{7} \\
X_{6}=\left(Y_{1} Y_{7}\right)^{-1}=Z_{8}^{-1} & Y_{7}=\left(X_{1} X_{6}\right)^{-1}=Z_{9}^{-1} & Z_{9}=X_{1} X_{6}=Y_{7}^{-1}
\end{array}
$$

The birational maps $\widehat{\theta}_{i}: \widetilde{U}_{i} \rightarrow Y$ that resolve the singularities of $Y$ are 
described by the pull back of the generators of the ring of $G_{x}$-invariant functions $\left(T_{1}, T_{2}, T_{3}, T_{4}, T_{5}\right)$ :

$$
\begin{array}{lll}
\widehat{\theta}_{1}^{*}\left(T_{1}\right)=X_{1}, & \widehat{\theta}_{2}^{*}\left(T_{1}\right)=Y_{1}, & \widehat{\theta}_{3}^{*}\left(T_{1}\right)=Z_{8} Z_{9}, \\
\widehat{\theta}_{1}^{*}\left(T_{2}\right)=X_{4} X_{6}, & \widehat{\theta}_{2}^{*}\left(T_{2}\right)=Y_{2}, & \widehat{\theta}_{3}^{*}\left(T_{2}\right)=Z_{2}, \\
\widehat{\theta}_{1}^{*}\left(T_{3}\right)=X_{3}, & \widehat{\theta}_{2}^{*}\left(T_{3}\right)=Y_{5} Y_{7}, & \widehat{\theta}_{3}^{*}\left(T_{3}\right)=Z_{3}, \\
\widehat{\theta}_{1}^{*}\left(T_{4}\right)=X_{4}, & \widehat{\theta}_{2}^{*}\left(T_{4}\right)=Y_{1} Y_{2} Y_{7}, & \widehat{\theta}_{3}^{*}\left(T_{4}\right)=Z_{2} Z_{8}, \\
\widehat{\theta}_{1}^{*}\left(T_{5}\right)=X_{1} X_{3} X_{6}, & \widehat{\theta}_{2}^{*}\left(T_{5}\right)=Y_{5}, & \widehat{\theta}_{3}^{*}\left(T_{5}\right)=Z_{3} Z_{9} .
\end{array}
$$

The point $O_{14,25,36}$ corresponds to the origin in $Y$ : let us study $\widehat{\theta}_{i}^{-1}(0)$

$$
\begin{gathered}
\widehat{\theta}_{1}^{-1}(0)=\left(0,0,0, t_{1}\right) \cong \mathrm{C}, \quad \widehat{\theta}_{2}^{-1}(0)=\left(0,0,0, u_{1}\right) \cong \mathrm{C}, \\
\widehat{\theta}_{3}^{-1}(0)=\left(0,0, t_{2}, u_{2}\right) \cong \mathrm{C} \cup \mathrm{C}
\end{gathered}
$$

where $t_{1}, u_{1}, t_{2}, u_{2} \in \mathrm{C}$ and $t_{2} u_{2}=0$.

In particular the fiber $\widehat{\theta}_{3}^{-1}(0)$ is isomorphic to the union of two copies of C that have a common point $(0,0,0,0) \in \widetilde{U}_{3}$. Moreover by the gluing (20), $t_{1}, t_{2} \in \mathbb{C}$ give a cover of $\mathbb{P}^{1}(\mathbb{C})$, just like $u_{1}, u_{2} \in \mathbb{C}$.

In conclusion the resolution of $O_{14,25,36}$ in $X^{S}(221111) / G$ is determined by the union of two copies of $\mathrm{P}^{1}(\mathrm{C})$ that have a common point

$$
\widehat{\theta}^{-1}\left(O_{14,25,36}\right) \cong \mathbb{P}^{1}(\mathrm{C}) \cup \mathbb{P}^{1}(\mathrm{C}) \text { with a common point. }
$$

Let us calculate the resolutions of the three singular curves $C_{14}, C_{25}, C_{36}$ that meet in $O_{14,25,36}$ : we know that there is a correspondence between $C_{i j}, C_{h l}, C_{k n}$ and the three lines $s_{3}=\{(0,0, t, 0,0)\}, s_{2}=\{(0, t, 0,0,0)\}$, $s_{1}=\{(t, 0,0,0,0)\}$ of $Y$. Now let us calculate the fiber of a "generic" point of each line $s_{j}$, for the maps $\widehat{\theta}_{i}$. thus

Let $\xi_{3} \in C_{14}: \widehat{\theta}_{1}^{-1}\left(\xi_{3}\right)=(0, t, 0, \tau), \widehat{\theta}_{2}^{-1}\left(\xi_{3}\right)=$ Imposs., $\widehat{\theta}_{3}^{-1}\left(\xi_{3}\right)=\left(0, t, \tau^{-1}, 0\right)$;

$$
\widehat{\theta}^{-1}\left(\xi_{3}\right) \cong \mathbb{P}^{1}(\mathrm{C}), \quad \forall \xi_{3} \in C_{14} \quad \xi_{3} \neq O_{i j, h l, k n}
$$

In the same way for $\xi_{2} \in C_{25}$ and $\xi_{1} \in C_{36}, \xi_{1}, \xi_{2} \neq O_{i j, h l, k n}$ we obtain:

$$
\widehat{\theta}^{-1}\left(\xi_{2}\right) \cong \mathbb{P}^{1}(\mathbb{C}), \quad \widehat{\theta}^{-1}\left(\xi_{1}\right) \cong \mathbb{P}^{1}(\mathbb{C}) .
$$

In conclusion the map

$$
\widehat{\theta}: X^{S}(\widehat{m}) / G=\left(\mathbb{P}^{2}\right)^{6}(221111) / G \longrightarrow\left(\mathbb{P}^{2}\right)^{6}(222111) / / G=X^{S S}(m) / / G
$$


determines the quotient $X^{S}(\widehat{m}) / G$ : in fact $\widehat{\theta}$ is an isomorphism over

$$
X^{S}(\widehat{m}) / G \backslash\left(\bigcup_{\xi \in S} \widehat{\theta}^{-1}(\xi)\right) \stackrel{\sim}{\longrightarrow} X^{S}(m) / G,
$$

where $S=\left\{\xi \in X^{S S S}(m) / / G\right\}$.

Then the map $\widehat{\theta}$ is a contraction of subvarieties over $\bigcup_{\xi \in S} \widehat{\theta}^{-1}(\xi)$ :

- if $\xi \in C_{i j}$, then $\widehat{\theta}^{-1}(\xi)=\mathbb{P}^{1}(\mathbb{C})$;

- if $\xi=O_{i j, h l, k n}$, then $\widehat{\theta}^{-1}(\xi)=\mathbb{P}^{1}(\mathbb{C}) \cup \mathbb{P}^{1}(\mathbb{C})$, with a common point;

- if $\xi=O_{456}$, then $\widehat{\theta}^{-1}(\xi)=\mathbb{P}^{3}(\mathbb{C})$.

Acknowledgments. The results of this paper were obtained during my Ph.D. studies at University of Bologna and are also contained in my thesis [8] with the same title. I would like to express deep gratitude to my supervisor prof. Luca Migliorini, whose guidance and support were crucial for the successful completion of this project. Moreover I am deeply grateful to the referee for helpful comments and corrections.

\section{REFERENCES}

[1] D. Cox, The homogeneous coordinate ring of a toric variety, J. Alg. Geom., 4 (1995), pp. 17-50.

[2] D. Cox, Toric variety and Toric resolutions, Resolution of Singularities (H. Hauser, J. Lipman, F. Oort, A. Quiros, eds), Birkhäuser (Basel-Boston-Berlin, 2000), pp. 259-284.

[3] I. V. Dolgachev, Lectures on Invariant Theory, Cambridge University Press, Lecture Note Series 296, 2003.

[4] I. V. Dolgachev - Y. Hu, Variations of geometric invariant theory quotients, Publ. Math. IHES, 87 (1998), pp. 5-51.

[5] J. M. DRÉzet, Luna's Slice Theorem, Notes for a course in Algebraic group actions and quotients at Wykno (Poland, Sept. 3-10, 2000).

[6] W. Fulton, Introduction to Toric Varieties, Princeton Univ. Press, 1993.

[7] R. Hartshorne, Algebraic Geometry, Springer-Verlag, GTM 52 (1977).

[8] F. InCEnsi, Quozienti GIT di prodotti di spazi proiettivi, $\mathrm{PhD}$ Thesis (2006).

[9] D. Luna, Slices Étales, Mém. Bull. Soc. Math de France, 33 (1973), pp. 81-105.

[10] D. Mumford - J. Fogarty - F. Kirwan, Geometric Invariant Theory, Springer-Verlag, 1994.

[11] M. ThadDEus, Geometric invariant theory and flips, Jour. Amer. Math. Soc., 9 (1996), pp. 691-723.

[12] C. WALTER, Variation of quotients and étale slices in geometric invariant theory, Notes for a course in Algebra and Geometry at Dyrkolbotn, Norway (Dec. 4-9, 1995).

Manoscritto pervenuto in redazione il 12 febbraio 2008. 